\title{
3D inversion of full gravity gradient tensor data in spherical coordinate system using local north-oriented frame
}

\author{
Yi Zhang ${ }^{1}$, Yulong Wu ${ }^{1 *}$, Jianguo Yan², Haoran Wang ${ }^{3,4}$, J. Alexis P. Rodriguez ${ }^{5}$ and Yue Qiu ${ }^{6}$
}

\begin{abstract}
In this paper, we propose an inverse method for full gravity gradient tensor data in the spherical coordinate system. As opposed to the traditional gravity inversion in the Cartesian coordinate system, our proposed method takes the curvature of the Earth, the Moon, or other planets into account, using tesseroid bodies to produce gravity gradient effects in forward modeling. We used both synthetic and observed datasets to test the stability and validity of the proposed method. Our results using synthetic gravity data show that our new method predicts the depth of the density anomalous body efficiently and accurately. Using observed gravity data for the Mare Smythii area on the moon, the density distribution of the crust in this area reveals its geological structure. These results validate the proposed method and potential application for large area data inversion of planetary geological structures.
\end{abstract}

Keywords: Gravity gradient tensor, Inversion, Density distribution, Spherical coordinate system, Local north-oriented frame

\section{Introduction}

The gravity gradient tensor (GGT) is the second derivative of the gravity potential. Compared to the general gravity field $T_{z}$, GGT contains nine components and has much higher resolution in inverting the spatial position of target anomaly bodies, which means it will offer more information to better understand the interior structure of the earth or some other planets ( $\mathrm{Li}$ 2001; Bouman et al. 2016).

In 1886, a torsion balance gradiometer was first developed by Lorand Eotvos, becoming a useful tool for mining and hydrocarbon exploration (Pedersen and Rasmussen 1990; Bell and Hansen 1998). 3D inversion of GGT data was originally introduced by Vasco (1989) and Vasco and Taylor (1991), who focused on the covariance and resolution measures of the solution appraisal. More recently, several algorithms were developed to inverse GGT data (e.g., Li 2001; Zhdanov et al. 2004; Uieda and

\footnotetext{
*Correspondence: yunlongwu@gmail.com

${ }^{1}$ Key Laboratory of Earthquake Geodesy, Institute of Seismology, China Earthquake Administration, 48 Hongshan Side Road, Wuhan 430071, Hubei, China

Full list of author information is available at the end of the article
}

Barbosa 2012; Oliveira and Barbosa 2013; Martinez et al. 2012; Geng et al. 2015; Meng 2016). The differences among these algorithms are related to the choice of model object functions in the inversion procedure; all the model object functions can be retraced back to the inversion methods used in the inversion of the general gravity field (e.g., Last and Kubik 1983; Guillen and Menichetti 1984; Barbosa and Silva 1994; Li and Oldenburg 1996, 1998; Farquharson 2008).

In addition to the choice of model object functions, there is little agreement on best component of GGT for the inversion. $\mathrm{Li}$ (2001) combined five independent components excluding $T_{z z}$. Zhdanov et al. (2004) used the horizontal components $T_{x y}$ and $T_{u v}=\left(T_{x x}-T_{y y}\right) / 2$. Martinez et al. (2012) combined the horizontal components and $T_{z z}$. Capriotti et al. (2015) employed a combination of the GGT and general gravity field $T_{z}$. Pilkington (2012) used an eigenvalue spectra method to evaluate the utility of combining different GGT components, preferring the $T_{z z}$ component, indicating that the source-model information is improved by adding more components only at close distance to the anomaly sources. Later, Pilkington (2013) used estimated parameter errors from parametric 
inversions, concluding that the $T_{z z}$ component gave the best performance, while the horizontal components $T_{x x}$ and $T_{y y}$ performed poorly. Paoletti et al. (2016) used a singular value decomposition (SVD) tool to analyze both synthetic data and gradiometer measurements. This research showed that the main factors controlling the reliability of the inversion are algebraic ambiguity (the difference between the number of unknowns and the number of available data points) and signal-to-noise ratio.

All these inversion methods mentioned are implemented in the Cartesian coordinate system (CCS). For small-scale problems such as mining or hydrocarbon exploration on earth, the target area is usually relatively small compared to the radius of the earth and can be considered as a flat surface, so inversion in CCS works fine and obtains reliable inversion results with high accuracy. However, for large-scale inversion problems, such as density imaging of the lunar mascon with the satellite gravity datasets, the target area of the lunar mascon was usually large and covered an area with hundreds or thousands of kilometers in both longitude and latitude directions. Moreover, the radius of the moon was relatively small $(1738 \mathrm{~km})$, and this meant the inversion area of the mascon was no longer a flat area; hence, inversion methods cannot ignore the influences of the lunar curvature. To deal with this problem, the inversion method in the SCS must be considered. Liang et al. (2014) extended $\mathrm{Li}$ and Oldenburg's $(1996,1998)$ inversion method to the SCS using the general gravity field.

In the history of the Moon exploration, one of the most amazing discoveries was the concentrated areas of mass found on the near side of the moon (Muller and Sjogren 1968; Melosh et al. 2013; Freed et al. 2014). These concentrated areas of mass, referred to as mascons, usually have a positive gravity anomaly peak and surrounded by negative gravity anomalies with low geographical elevation. The knowledge of the interior density structure of mascons will help to understand its origin mechanism (Wang et al. 2015; Jansen et al. 2017). There has a significant improvement on lunar gravity field model development (Matsumoto et al. 2010; Yan et al. 2012), and the recent high solution gravity field model GL1500E derived from GRAIL mission (The Planetary Data System 2016) makes it possible to investigate the interior density structure of the mascons (Zuber et al. 2013a, b) using lunar gradient data.

The high-resolution lunar gradient data produced from GRAIL gravity was applied by Andrews-Hanna et al. (2014) to investigate the structure and evolution of the lunar Procellarum region. The fault geometry, thermal structure, and material content were considered to generate rectilinear patterns of the gradient data in this region.
A forward modeling method coupling with finite element method (FEM) was employed this work. GGT is the difference of gravity in different directions, which helps to remove the influence of the long-wave part in the lunar gravity, and it is easier to highlight the lunar gravity's shortwave effect and to get a better resolution for density imaging. In this work, we will focus on inversion of the lunar gradient data, which is different from the previous work (Andrews-Hanna et al. 2013).

The remainder of this paper is as follows: a brief introduction of this inversion method is presented in second section. Third section details two different models and experiments with synthetic GGT datasets and inversion of GGT observation datasets of moon. The interior density structure of the Mare Smythii mascon is discussed in fourth section. Finally, in fifth section we present the conclusions of this study.

\section{Methodology}

\section{GGT in the spherical coordinate system}

The two most commonly used frames in SCS are the geocentric spherical frame (GSF) and the local northoriented frame (LNOF). The GGT is expressed in terms of the second derivatives of the gravitational potential $U$ in the $r, \lambda$, and $\varphi$ directions of GSF, where $r, \lambda$, and $\varphi$ refer to the radial, longitude, and latitude, respectively (Eq. 1).

$T=\left[\begin{array}{ccc}T_{\lambda \lambda} & T_{\lambda \varphi} & T_{\lambda r} \\ T_{\varphi \lambda} & T_{\varphi \varphi} & T_{\varphi r} \\ T_{r \lambda} & T_{r \varphi} & T_{r r}\end{array}\right]=\left[\begin{array}{ccc}\frac{\partial^{2} U}{r^{2} \cos ^{2} \varphi \partial \lambda^{2}} & \frac{\partial^{2} U}{r^{2} \cos \varphi \partial \lambda \partial \varphi} & \frac{\partial^{2} U}{r \cos \varphi \partial \lambda \partial r} \\ \frac{\partial^{2} U}{r^{2} \cos \varphi \partial \lambda \partial \varphi} & \frac{\partial^{2} U}{r^{2} \partial \varphi^{2}} & \frac{\partial^{2} U}{r \partial \varphi \partial r} \\ \frac{\partial^{2} U}{r \cos \varphi \partial \lambda \partial r} & \frac{\partial^{2} U}{r \partial \varphi \partial r} & \frac{\partial^{2} U}{\partial r^{2}}\end{array}\right]$

In LNOF, where $z$ has the geocentric radial downward direction, $x$ points to the north, and $y$ is directed to the east with a right-handed system, relationship between the LNOF and GSF can be described as in Eq. (2) (Reed 1973; Petrovskaya and Vershkov 2006).

$$
\begin{aligned}
T_{x x} & =\frac{1}{r} T_{r}+\frac{1}{r^{2}} T_{\varphi \varphi} \\
T_{x y} & =\frac{1}{r^{2} \cos \varphi} T_{\lambda \varphi}+\frac{\sin \varphi}{r^{2} \cos ^{2} \varphi} T_{\lambda} \\
T_{x z} & =\frac{1}{r^{2}} T_{\varphi}-\frac{1}{r} T_{r \varphi} \\
T_{y y} & =\frac{1}{r} T_{r}+\frac{1}{r^{2} \cot \varphi} T_{\varphi}+\frac{1}{r^{2} \cos ^{2} \varphi} T_{\lambda \lambda} \\
T_{y z} & =\frac{1}{r^{2} \cos \varphi} T_{\lambda}-\frac{1}{r \cos \varphi} T_{r \lambda} \\
T_{z z} & =T_{r r}
\end{aligned}
$$

In this paper, we choose to use LNOF, which will not be singular when calculating GGT components from a gravity spherical harmonics model (Eshagh 2008, 2010) and because the GGT is symmetric and the trace of the 
GGT equals zero; hence, there are only five independent components.

\section{Forward modeling}

Theoretically, forward modeling is the basis of the inversion, as it forms the relationship between the model and data space. The forward modeling method we use here was developed by Asgharzadeh et al. (2007) for calculating a gravity field and its gradients in the SCS with LNOF. This method makes use of the Gauss-Legendre quadrature integration for numerically modeling theoretical gravity effects caused by the tesseroids (Anderson 1976; Heck and Seitz 2006) (Fig. 1).

According to Asgharzadeh et al. (2007), each component of the GGT can form its own relationship between the model and dataset and can be described as:

$$
T_{i j}=G_{i j} m \quad i, j=x, y, z
$$

In Eq. (3), $m$ and $G_{i j}$ refer to the model and kernel matrix, respectively.

To make full use of the GGT dataset, we adopt the new kernel function $G$ as the linear combination of the five independent components of GGT:

$$
G_{s}=\sum_{i, j}^{x, y, z}\left(k_{i j} G_{i j}\right)
$$

$k_{i j}$ here refers to the weighting factor of each component, and it can be considered as the data accuracies (or the reliabilities) of each component.

Due to the relationship of $T_{x x}+T_{y y}+T_{z z}=0$, the linear combination of the components $T_{x x}$ and $T_{y y}$ can be described by the vertical components $T_{z z}$; hence, we do not employ the components $T_{x x}$ and $T_{y y}$ in Eq. (4).

Similarly, the GGT dataset $d_{s}$ can be also adopted into the linear combination of the independent components:

$$
d_{s}=T_{s}=\sum_{i, j}^{x, y, z}\left(k_{i j} T_{i j}\right)
$$

For each independent component $T_{i j}$, the error standard deviation is $\sigma_{i j}$. According to error theory, the error standard deviation of the GGT will be:

$$
\sigma=\sqrt{\sum_{i, j}^{x, y, z}\left(k_{i j} \sigma_{i j}\right)^{2}+\operatorname{Cov}}
$$

In Eq. (6), Cov represents the sum of the error covariance of all components. On the assumption that the error of the GGT follows the Gaussian random distribution and one independent component has no connection with each other, then $\operatorname{Cov}=0$.

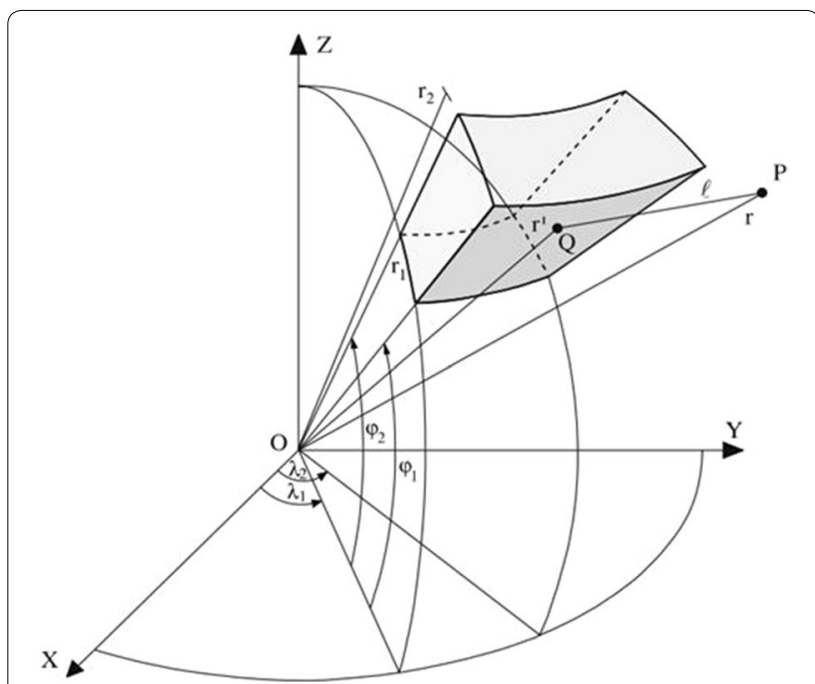

Fig. 1 Geometry of the tesseroid (Heck and Seitz 2006), point $O$ is the original point of the SCS; $r_{1}$ and $r_{2}, \lambda_{1}$ and $\lambda_{2}$, as well as $\varphi_{1}$ and $\varphi_{2}$ are the boundaries in radial, longitude, and latitude directions, respectively. Point $Q$ here denotes the geometric center of the tesseroid, and point $P$ is an observation data point

\section{Inversion method}

In general, because of an insufficient observed dataset, the multiple solutions problem becomes a serious issue for the 3D gravity inversion. To deal with the problem, a suitable model objective function is required.

$\mathrm{Li}$ and Oldenburg (1996) and Li (2001) designed a model objective function with a maximum smoothing method; however, this model objective function is only suitable for CCS. Liang et al. (2014) extended it to the SCS using the spherical derivative operators:

$$
\begin{aligned}
\phi_{m}(m)= & \alpha_{s} \int_{V}\left[w(r)\left(m-m_{\mathrm{ref}}\right)\right]^{2} \mathrm{~d} v \\
& +\alpha_{r} \int_{V}\left[\frac{\partial w(r)\left(m-m_{\mathrm{ref}}\right)}{\partial r}\right]^{2} \mathrm{~d} v \\
& +\alpha_{\varphi} \int_{V}\left[\frac{\partial w(r)\left(m-m_{\mathrm{ref}}\right)}{r \partial \varphi}\right]^{2} \mathrm{~d} v \\
& +\alpha_{\lambda} \int_{V}\left[\frac{\partial w(r)\left(m-m_{\mathrm{ref}}\right)}{r \cos \varphi \partial \lambda}\right]^{2} \mathrm{~d} v
\end{aligned}
$$

The model object function of Eq. (7), also called stabilized function, was initially introduced by Backus and Gilbert $(1967,1968,1970)$ to solve ill-posed inverse problems. It can be divided into two parts: the first item of Eq. (7) is the smallest model between recovered and reference model, and the last three items are the smoothest model between recovered and reference model in 
Table 1 3D mesh and dataset for inversion

\begin{tabular}{|c|c|c|c|c|c|}
\hline \multirow[t]{2}{*}{ Direction } & \multirow[t]{2}{*}{ Inversion range } & \multicolumn{2}{|l|}{ Model } & \multicolumn{2}{|l|}{ Data } \\
\hline & & Grid size & Grid number & Data size & Data number \\
\hline Longitude & $30.0^{\circ}-40.0^{\circ}$ & $0.25^{\circ}$ & 40 & $0.25^{\circ}$ & 41 \\
\hline Latitude & $30.0^{\circ}-40.0^{\circ}$ & $0.25^{\circ}$ & 40 & $0.25^{\circ}$ & 41 \\
\hline Depth/elevation & $0-100$ km & $5 \mathrm{~km}$ & 20 & $0.5 \mathrm{~km}$ & \\
\hline
\end{tabular}

longitudinal, latitudinal, and radial directions, respectively. In Eq. (7), $m$ and $m_{\text {ref }}$ refer to the recovered and reference model, respectively. $\alpha_{i}(i=s, r, \lambda, \varphi)$ are length scales, which control the balance of the smoothness versus smallness for the whole model, $\alpha_{s}$ for smallness, and $\alpha_{r}, \alpha_{\lambda}$, and $a_{\varphi}$ for smoothness (Oldenburg and Li 2005; Williams 2008). In practice, $\alpha_{s}$ usually can be assigned to a value of 1.0 or other suitable value; however, different to those in CCS (Williams 2008), $\alpha_{\lambda}$, and $a_{\varphi}$ are variable because of the different tesseroid body sizes along the radial direction. $w(r)$ here represents the depth weighting function, and it can be used to avoid the skin effect in the inversion ( $\mathrm{Li}$ and Oldenburg 1996; Li 2001). The depth weighting functions match the decay of the gravity or magnetic kernel functions, and they are in proportion to $1 / r^{2}$ in gravity and $1 / r^{3}$ in magnetic inversion problem. Without them, the inversion will get results concentrated on the surface of the target area ( $\mathrm{Li} 2001)$. Unlike the uniform prism cells in CCS, the tesseroid cells become smaller along the radial direction from surface to the core, so it must rescale them into the same level. Liang's et al. (2014) main contribution is the modification of the depth weighting function in SCS by rescaling the cell volume to the surface (see Additional file 1).

$$
w^{2}(r)=\frac{r^{2}}{r_{0}^{2}(H+R-r)^{2}}
$$

In Eq. (8), $R$ is the radius of the reference sphere and $H$ is the average height of observed dataset above the reference sphere, while $r_{0}$ and $r$ are the radial distance of the surface and for computing tesseroid cells, respectively.

In addition, geological and geophysical constraints play an important role in the gravity inversion. The geological and geophysical constraints are varied, and they can be classified into two different kinds: (1) geometry constraints like structure boundaries, orientations, and locations information; (2) physical property constraints such as surface material content and information from drill holes. All these constraints can be described as the function of the physical property and positions. In our inversion method, as we divided the subspace into different tesseroid cells, the constraints become the function of the physical property and index number of the tesseroid cells.

Different from the model objective function, which aims to solve the non-uniqueness in ill-posed inverse problems, the purpose of using a prior geological and geophysical information during the inversion is to improve the inversion result. In this paper, we use the Lagrangian multipliers method, introduced by Zhang et al. (2015), to fit for the different prior geological or geophysical information during the inversion procedure; the additional penalty function of the density bound constraints makes the recovered model more reliable.

\section{Examples of synthetic GGT data}

In this section, we will give two examples of the artificial synthetic model used to validate our inversion method.

\section{Single model}

Table 1 shows the 3D mesh and dataset setting for inversion. The reference radius we use is $1738 \mathrm{~km}$, which is the same as the mean radius of the moon.

The artificial synthetic model we use here is a horizontal spherical prism body with constant residual density of $0.5 \mathrm{~g} / \mathrm{cm}^{3}$ and covers an area of $33^{\circ}-37^{\circ}$ in both the longitude and latitude directions as well as $1648-1698 \mathrm{~km}$ in radial direction. The background density was set to $0 \mathrm{~g} /$ $\mathrm{cm}^{3}$. Figure 2 shows the horizontal slice and vertical profile of the artificial synthetic model used for inversion.

In Figs. 3, 4, we showed the artificial theoretical datasets for all the components of the GGT. Considering the geometric relationships between the model mesh and the data grids in Table 1, we also obtained the kernel matrixes $G_{s}=G_{x y}+G_{x z}+G_{y z}+G_{z z}$ and the GGT dataset $d_{s}=d_{x y}+d_{x z}+d_{y z}+d_{z z}$, as shown in Fig. 4c.

Furthermore, independent Gaussian random noise was added to each component of the GGT dataset. The mean value of the Gaussian noise was zero, the standard deviation was approximately $1 \%$ of the difference between the maximum and minimum value of each component, and the artificial theoretical datasets with noise are shown in Figs. 5, 6.

In the inversion, we assigned a density constraint between 0 and $0.5 \mathrm{~g} / \mathrm{cm}^{3}$ to each inverted cell. The L-curve method (Calvetti et al. 2000) was used to search 
for the best Tikhonov regularization parameter (Tikhonov and Arsenin 1977), and the recovered model is shown in Fig. 7. Tikhonov regularization parameter here is the trade-off between the model objective function and data objective function (also named data misfit). The Tikhonov regularization parameter we chose is 10 , which is located at the corner of L-curve, but slightly offset to the right with a smaller data misfit (Zhang et al. 2015). The black lines in Fig. 7 indicate the boundary of the artificial synthetic model, and the recovered model was fitted well. The recovered model with minimum-structure inversions using $L_{2}$-norm measures usually has blurred boundaries, and if sharp boundaries and blocky features are needed, the non- $L_{2}$ inversions may provide an alternative method (Sun and Li 2014).
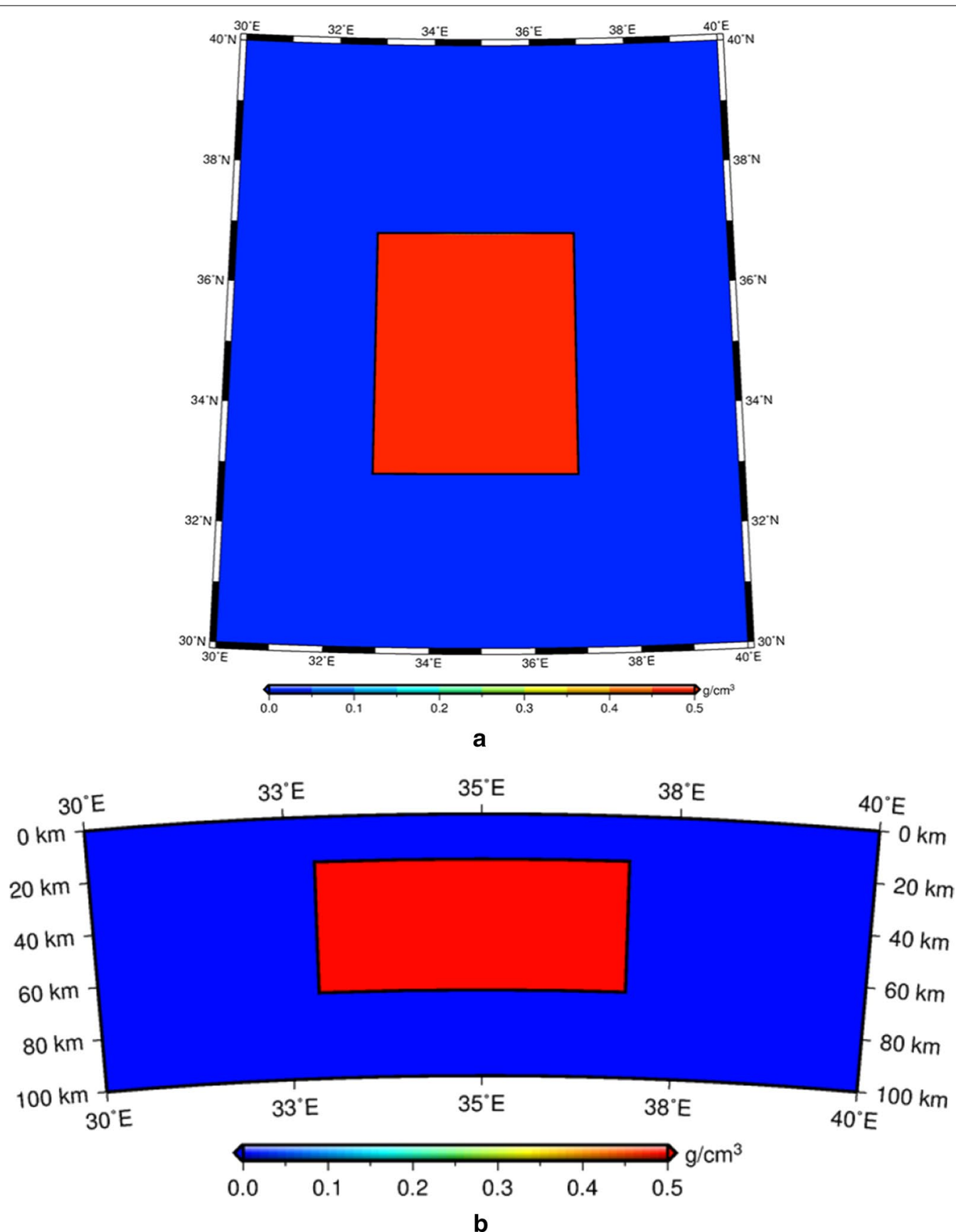

Fig. 2 Artificial synthetic model (a horizontal spherical prism body). a Horizontal slice of the model with a radial length of $1698 \mathrm{~km}$, b vertical profile of the model with latitude of $35^{\circ}$ 

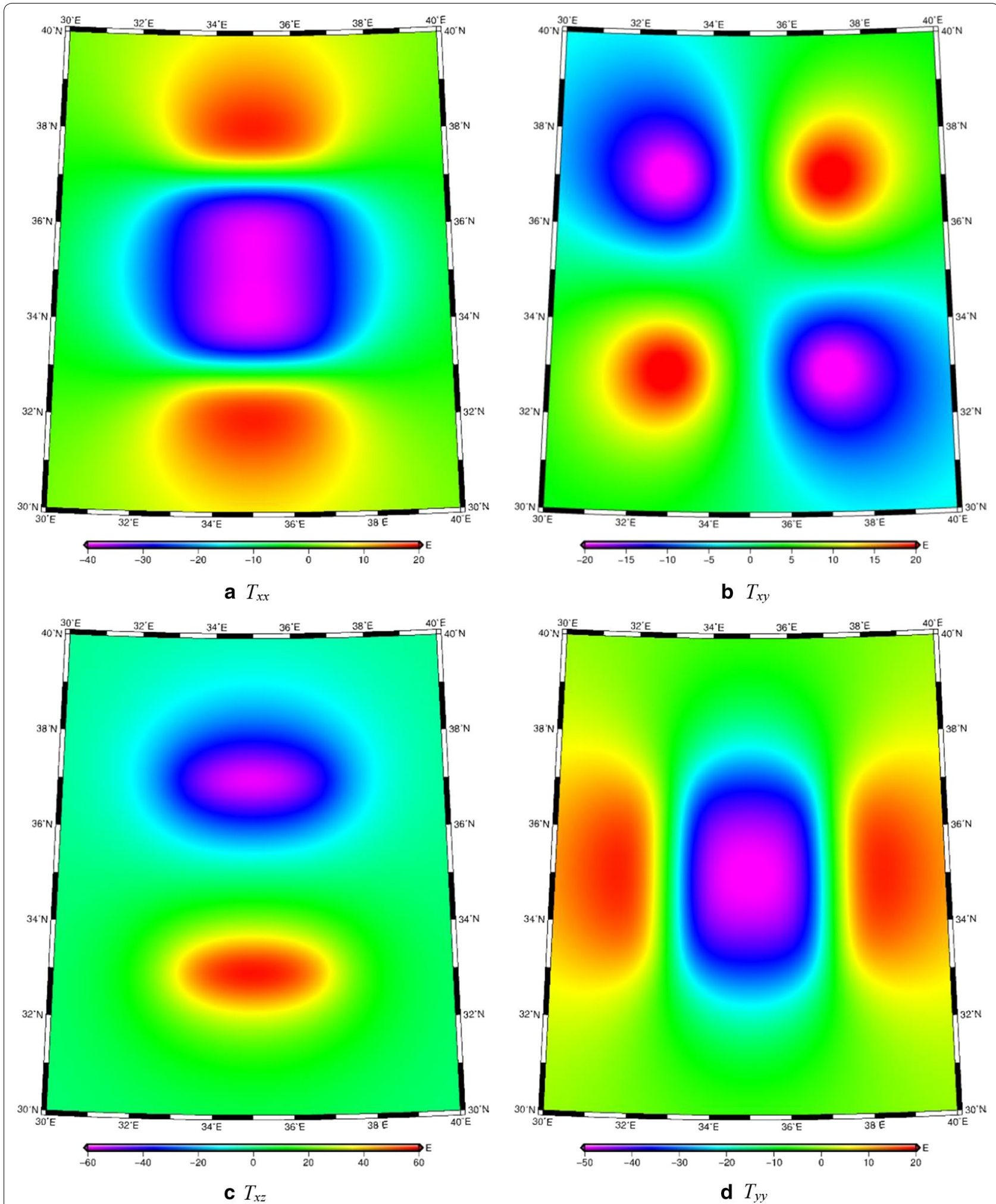

Fig. 3 Artificial theoretical datasets of the GGT for single model, $\mathbf{a}, \mathbf{b}, \mathbf{c}$ and $\mathbf{d}$ are $T_{x x}, T_{x y}, T_{x z}$ and $T_{y y}$ components, respectively 

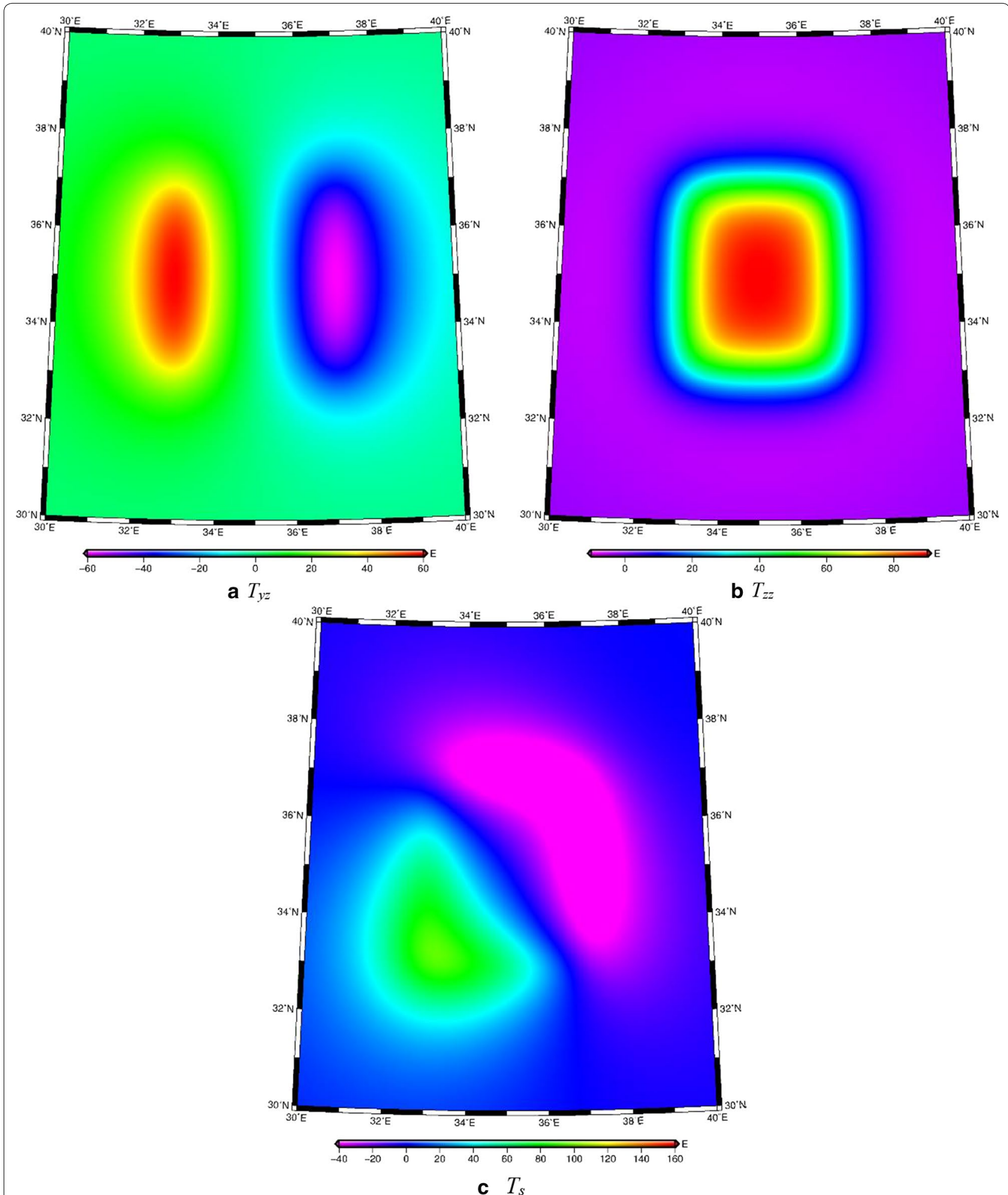

Fig. 4 Artificial theoretical datasets of the GGT for single model, $\mathbf{a}$ and $\mathbf{b}$ are $T_{y z}$ and $T_{z z}$ components of the GGT, respectively, and $\mathbf{c} T_{s}$ is the summation of $T_{x x^{\prime}} T_{x y y^{\prime}} T_{x z^{\prime}}, T_{y y^{\prime}} T_{y z}$ and $T_{z z}$ 

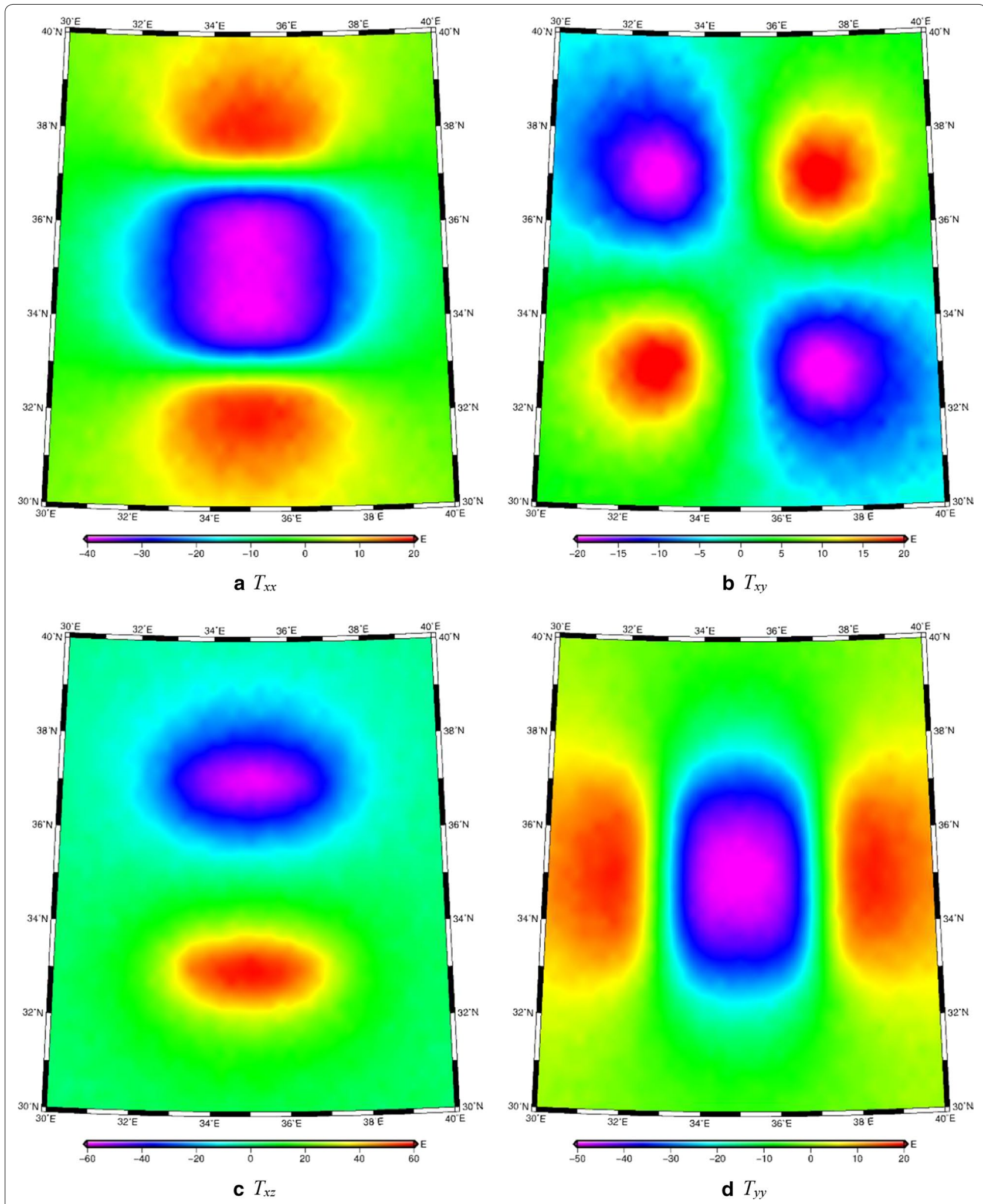

Fig. 5 Artificial theoretical datasets of the GGT with noise for single model, $\mathbf{a}, \mathbf{b}, \mathbf{c}$ and $\mathbf{d}$ are $T_{x x}, T_{x y}, T_{x z}$ and $T_{y y}$ component of the GGT, respectively 

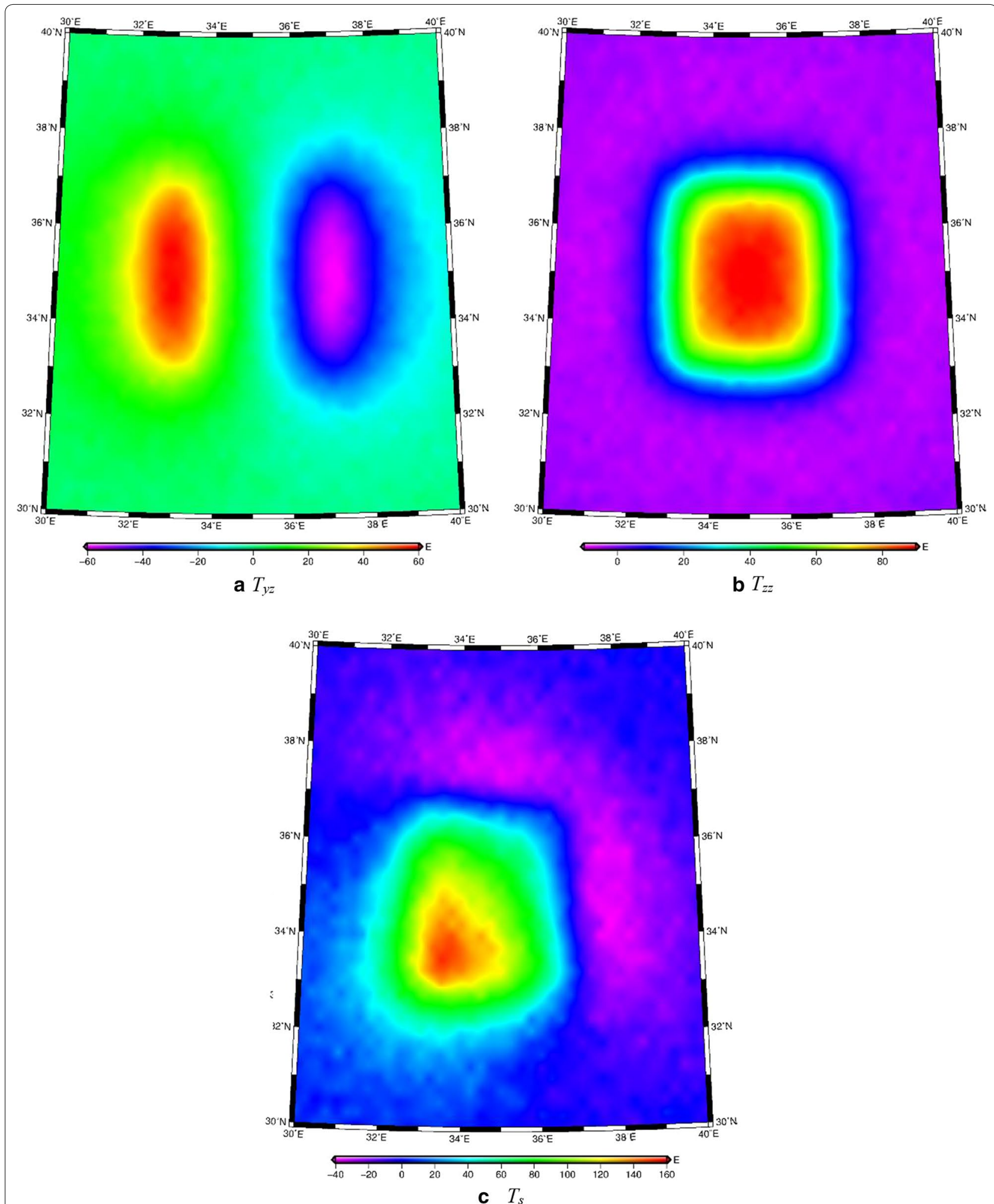

Fig. 6 Artificial theoretical datasets of the GGT with noise for single model, $\mathbf{a}$ and $\mathbf{b}$ are $T_{y z}$ and $T_{z z}$ components of the GGT, respectively, and $\mathbf{c} T_{s}$ is the summation of $T_{x x^{\prime}} T_{x y^{\prime}} T_{x z^{\prime}} T_{y y^{\prime}} T_{y z}$ and $T_{z z}$ 

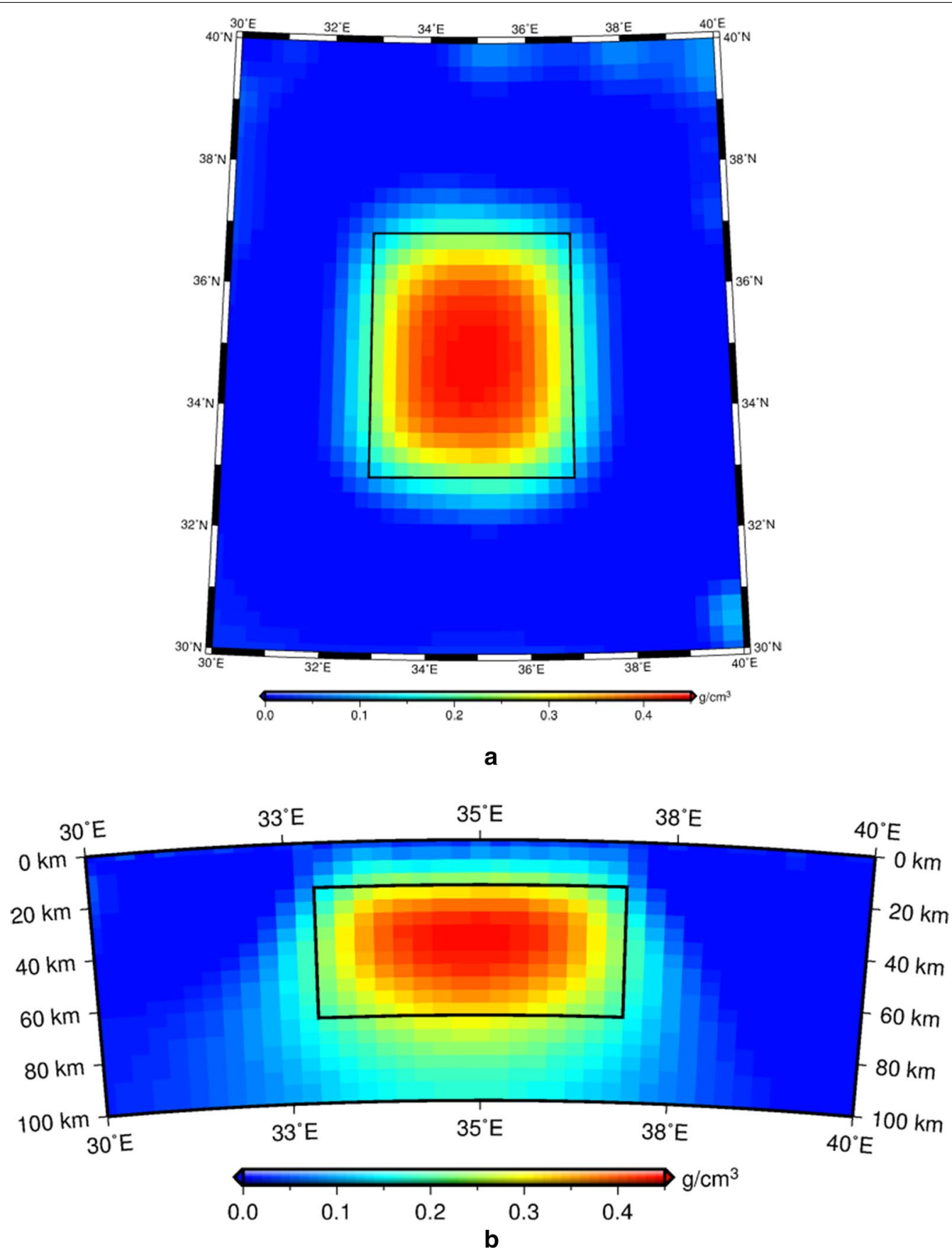

Fig. 7 Recovered density distribution for single model. a Horizontal slice of the recovered model with a radial length of $1698 \mathrm{~km}$, $\mathbf{b}$ vertical profile of the recovered model at the latitude of $35^{\circ}$

\section{Composite models}

Here we designed two composite models. One model covered an area of $34^{\circ}-36^{\circ}, 32^{\circ}-34^{\circ}$, and $1678-1718 \mathrm{~km}$ in latitude and longitude as well as radial direction, respectively, with a small residual density of $0.2 \mathrm{~g} / \mathrm{cm}^{3}$. The residual density of the other model was $0.7 \mathrm{~g} / \mathrm{cm}^{3}$, and its geological setting was $34^{\circ}-36^{\circ}, 36^{\circ}-38^{\circ}$, and $1658-1698 \mathrm{~km}$ in latitude and longitude as well as radial direction, respectively. The $3 \mathrm{D}$ mesh and dataset settings we used are shown in Table 1 . The horizontal slice and vertical profile of the artificial synthetic models are shown in Fig. 8. 

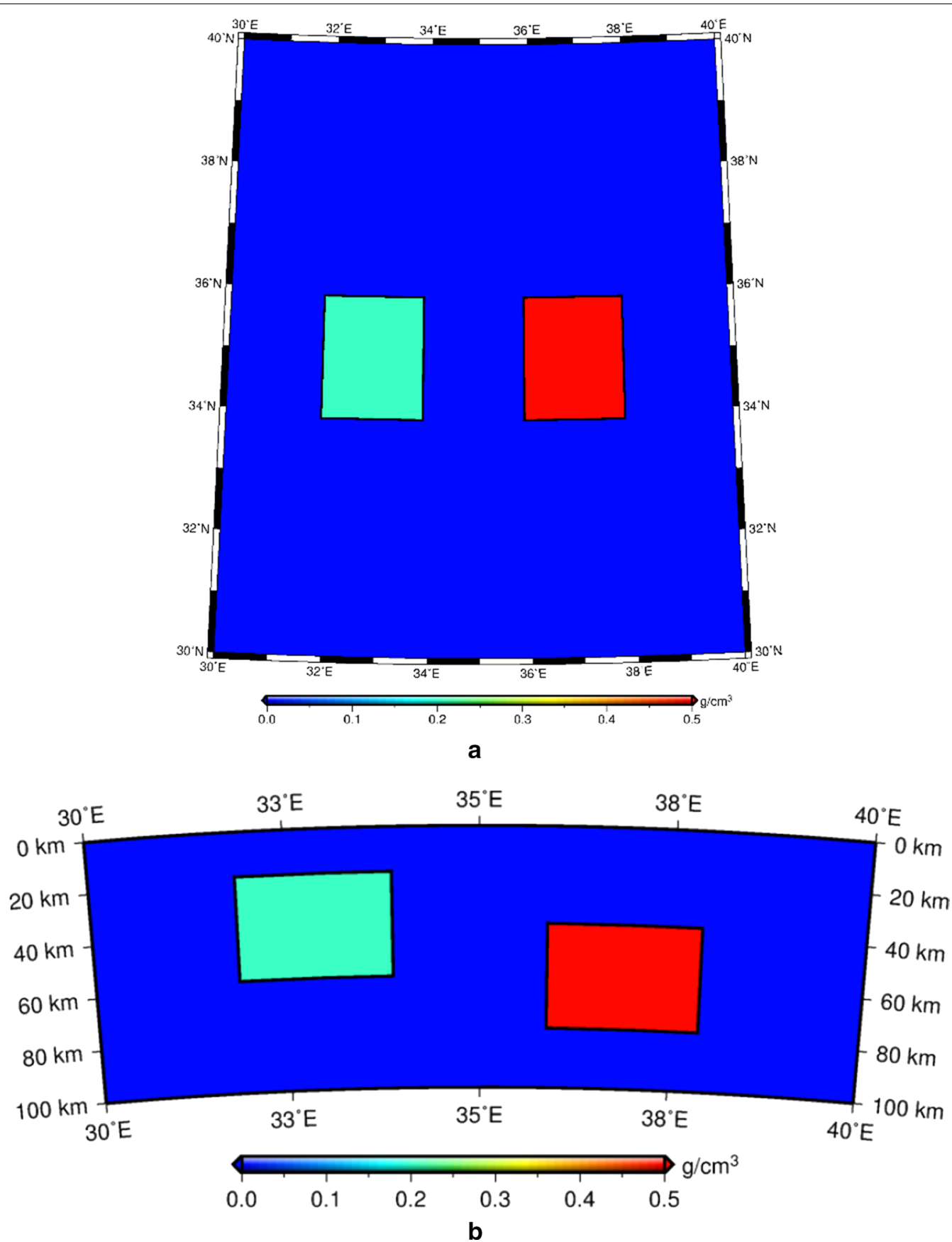

Fig. 8 Artificial synthetic models (two composite models). a Horizontal slice of the artificial synthetic model with a radial length of $1698 \mathrm{~km}, \mathbf{b}$ vertical profile of the artificial synthetic model with latitude of $35^{\circ}$

Similarly, we obtained the artificial theoretical datasets of all the components of the GGT (Figs. 9, 10), as well as the added $1 \%$ Gaussian random noise, as shown in Figs. 11, 12.

Using the same scheme, with a density bound of $0-0.7 \mathrm{~g} / \mathrm{cm}^{3}$, the recovered model was obtained, as in
Fig. 13; the recovered model was well fitted with the blank lines indicating the boundaries of the theoretical models.

Through the artificial theoretical models, we tested our inversion method in the SCS with LNOF. The results indicated that the method is possible to be employed in interior density structure model inversion. In the next 

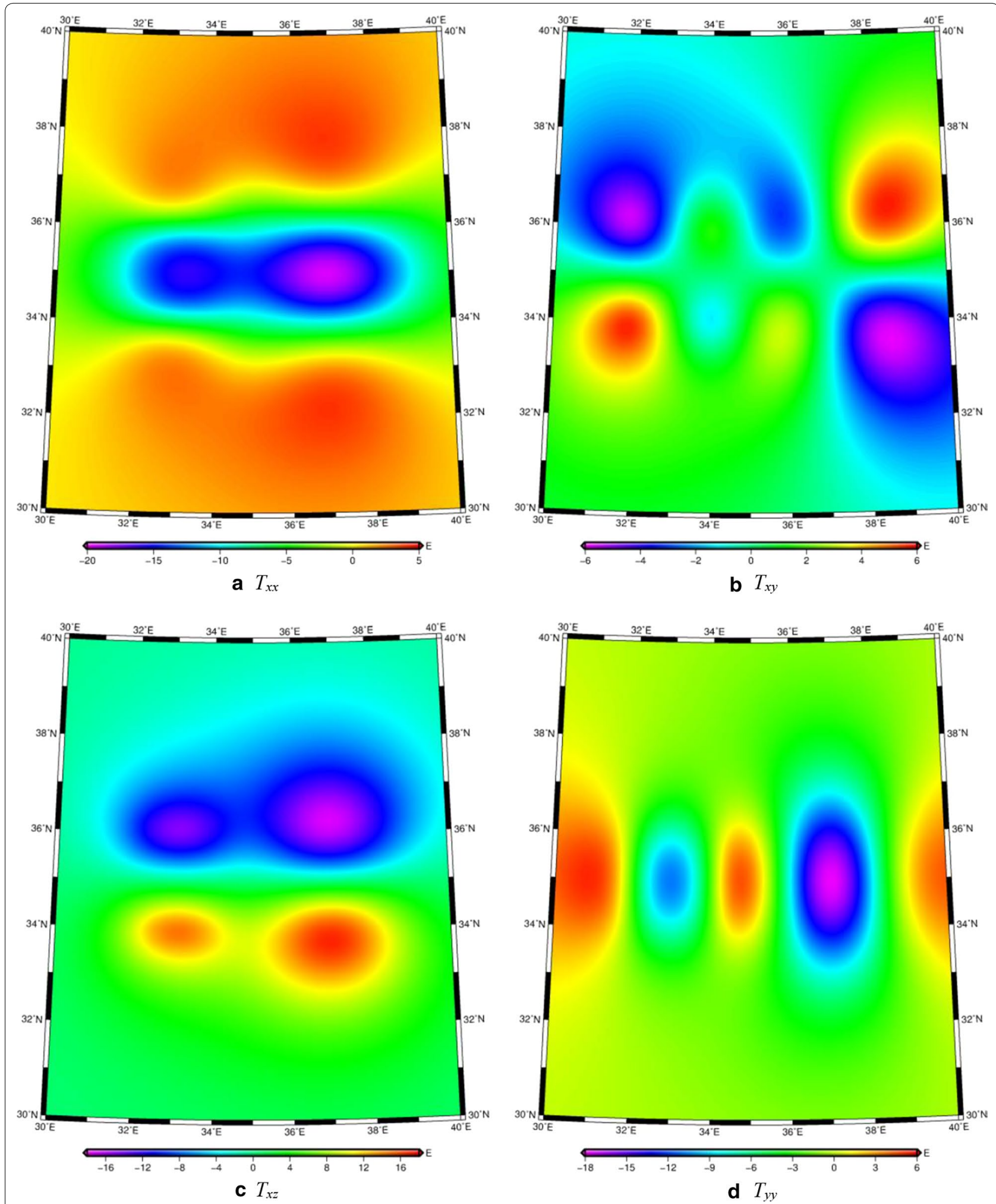

Fig. 9 Artificial theoretical datasets of the GGT for composite models, $\mathbf{a}, \mathbf{b}, \mathbf{c}$ and $\mathbf{d}$ are $T_{x x^{\prime}} T_{x y^{\prime}} T_{x z}$ and $T_{y y}$ components, respectively 

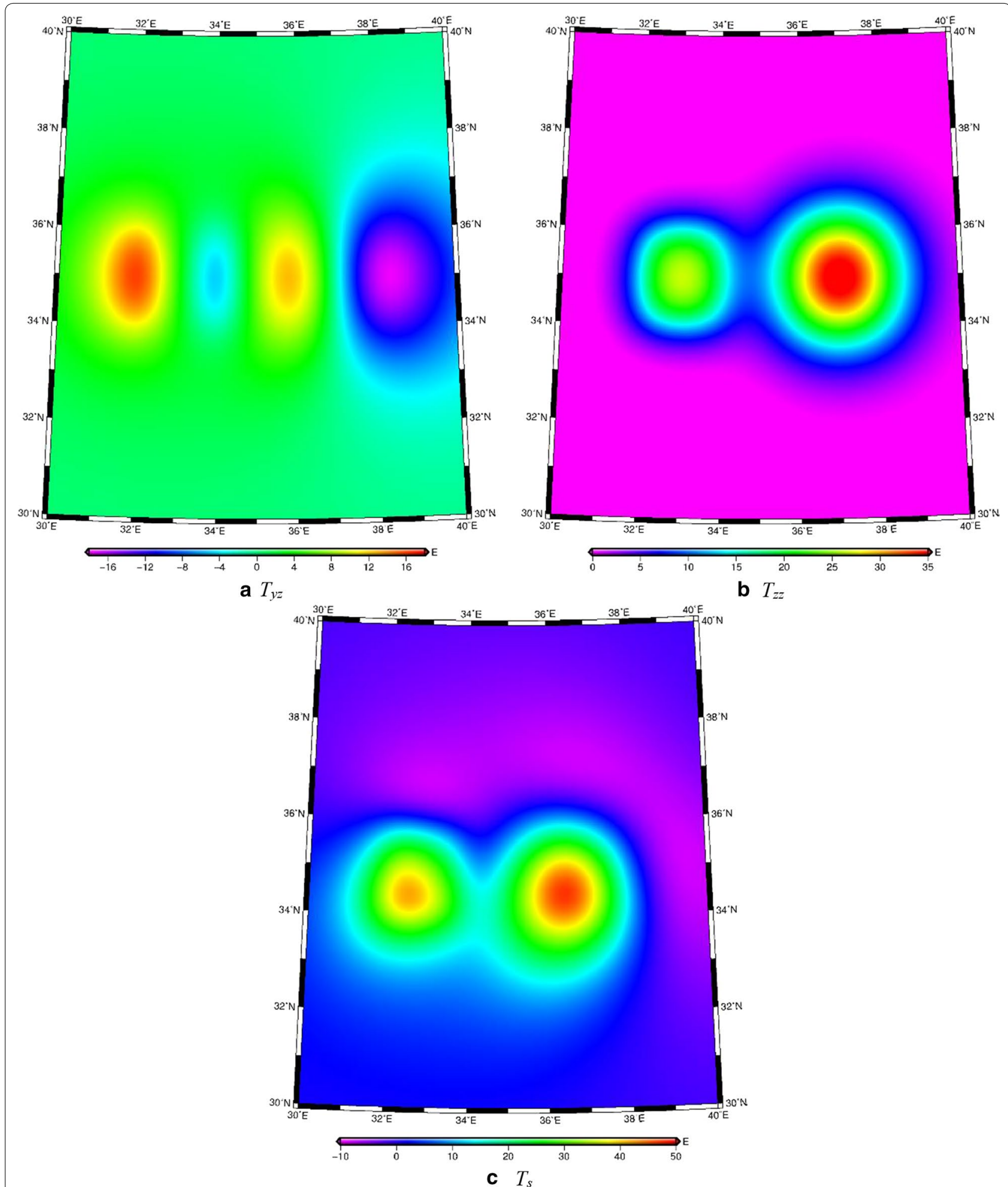

Fig. 10 Artificial theoretical datasets of the GGT for composite models, $\mathbf{a}$ and $\mathbf{b}$ are $T_{y z}$ and $T_{z z}$ components of the GGT, respectively, and $\mathbf{c} T_{s}$ is the summation of $T_{x x^{\prime}} T_{x y}, T_{x z^{\prime}} T_{y y^{\prime}} T_{y z}$ and $T_{z z}$ 

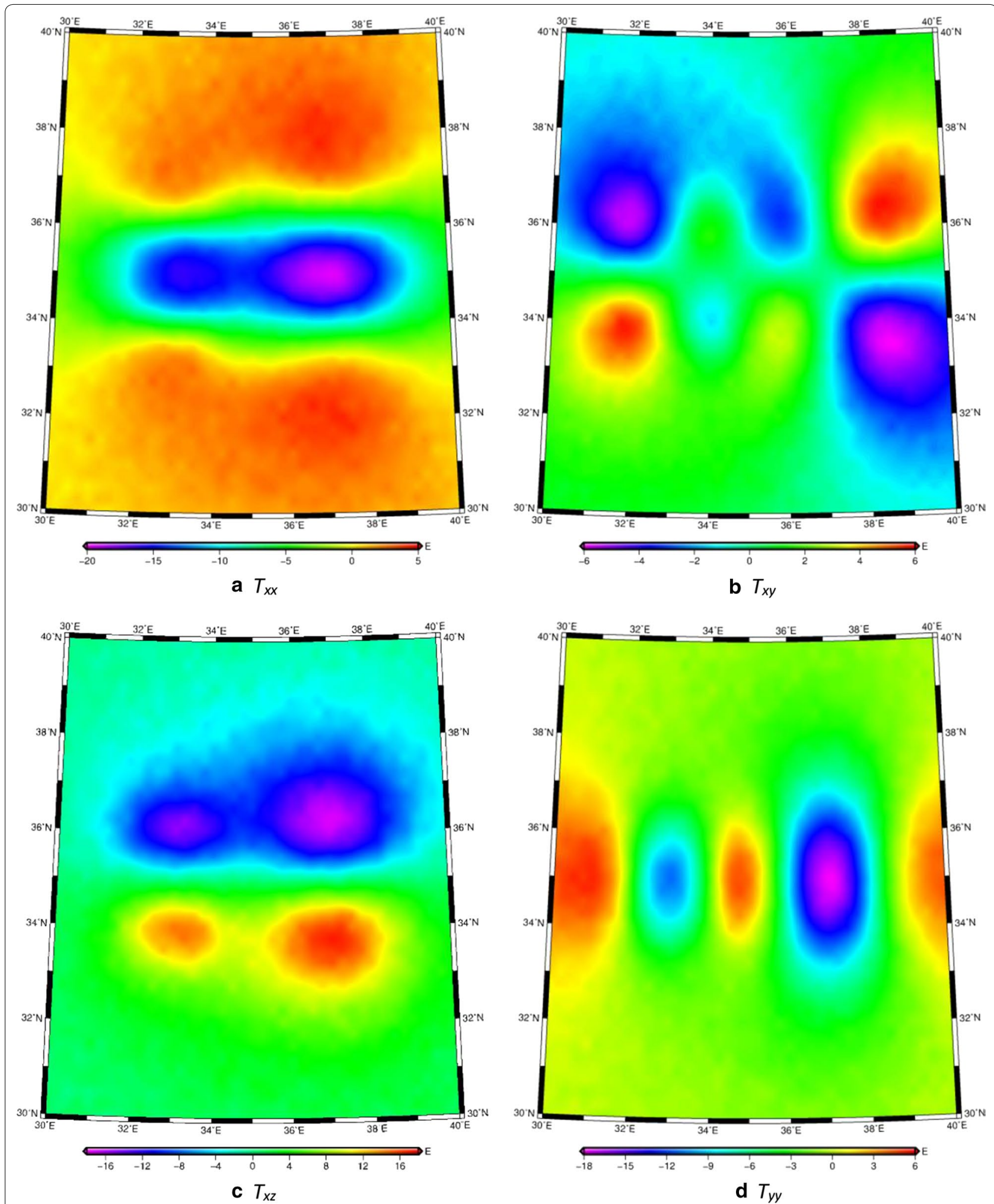

Fig. 11 Artificial theoretical datasets of the GGT with noise for composite models, $\mathbf{a}, \mathbf{b}, \mathbf{c}$ and $\mathbf{d}$ are $T_{x x \prime} T_{x y} T_{x z}$ and $T_{y y}$ component of the GGT, respectively 

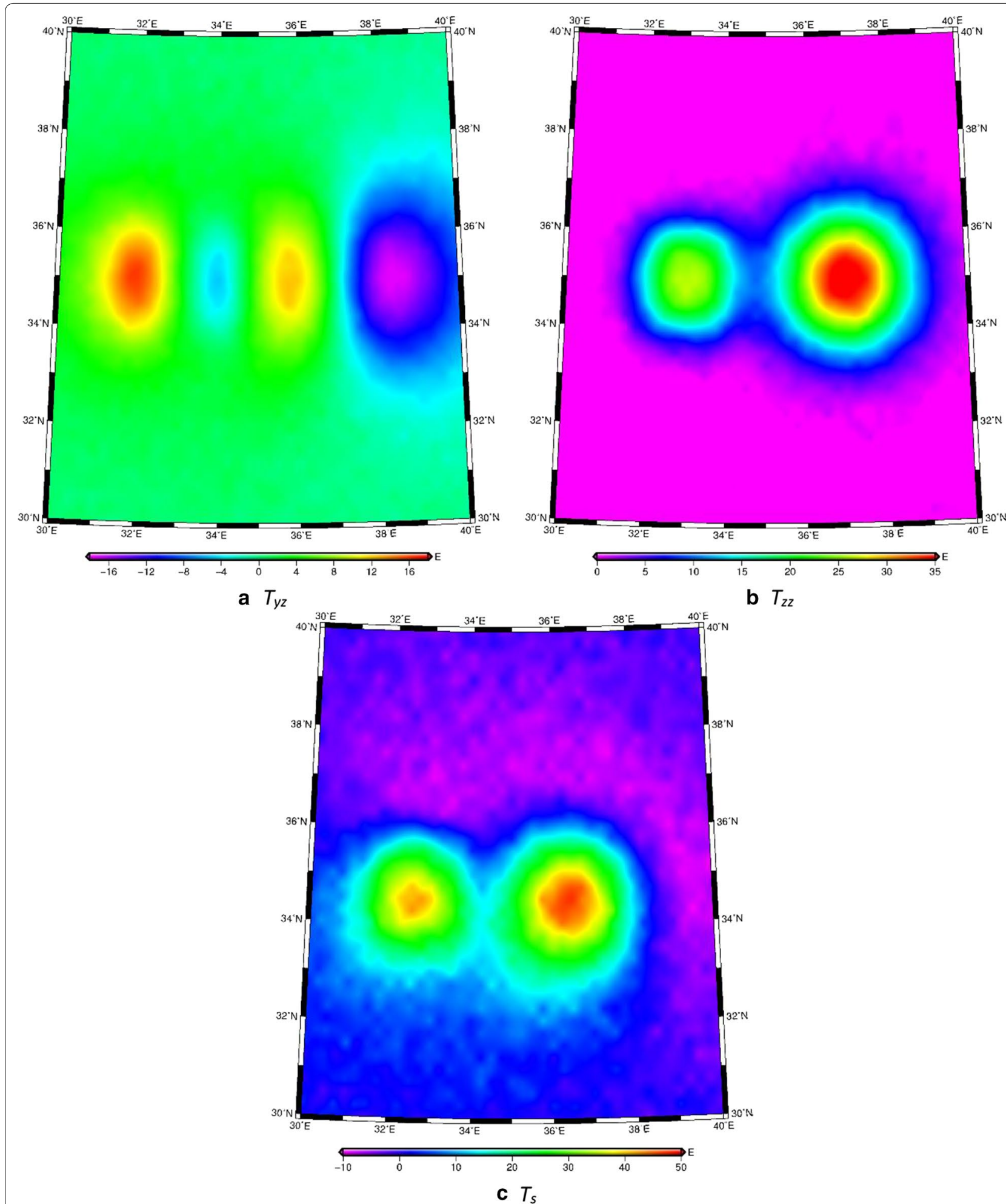

Fig. 12 Artificial theoretical datasets of the GGT with noise for composite models, $\mathbf{a}$ and $\mathbf{b}$ are $T_{y z}$ and $T_{z z}$ components of the GGT, respectively, and c $T_{s}$ is the summation of $T_{x x}, T_{x y}, T_{x z^{\prime}} T_{y y^{\prime}} T_{y z}$ and $T_{z z}$ 

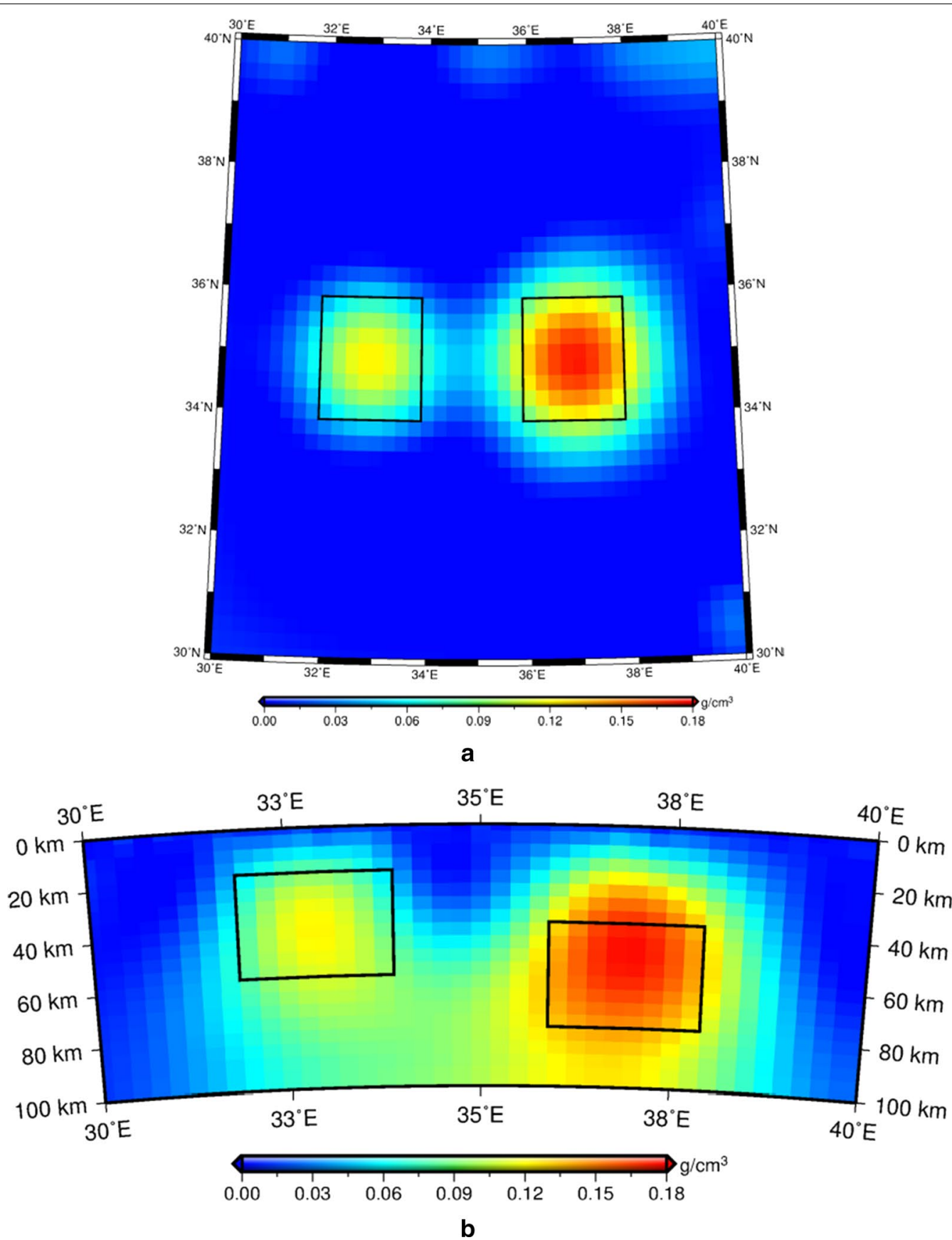

Fig. 13 Recovered density distribution for composite models. a Horizontal slice of the recovered model with a radial length of $1698 \mathrm{~km}$, b vertical profile of the recovered model at the latitude of $35^{\circ}$

Table 2 3D mesh and datasets for the inversion of Mare Smythii

\begin{tabular}{|c|c|c|c|c|c|}
\hline \multirow[t]{2}{*}{ Direction } & \multirow[t]{2}{*}{ Inversion range } & \multicolumn{2}{|l|}{ Model } & \multicolumn{2}{|l|}{ Data } \\
\hline & & Grid size & Grid number & Data size & Data number \\
\hline Longitude & $76.0^{\circ}-97.0^{\circ}$ & $0.25^{\circ}$ & 84 & $0.25^{\circ}$ & 85 \\
\hline Latitude & $-10.0^{\circ}-9.0^{\circ}$ & $0.25^{\circ}$ & 76 & $0.25^{\circ}$ & 77 \\
\hline Depth/elevation & $0-100$ km & $5 \mathrm{~km}$ & 20 & $0.5 \mathrm{~km}$ & \\
\hline
\end{tabular}



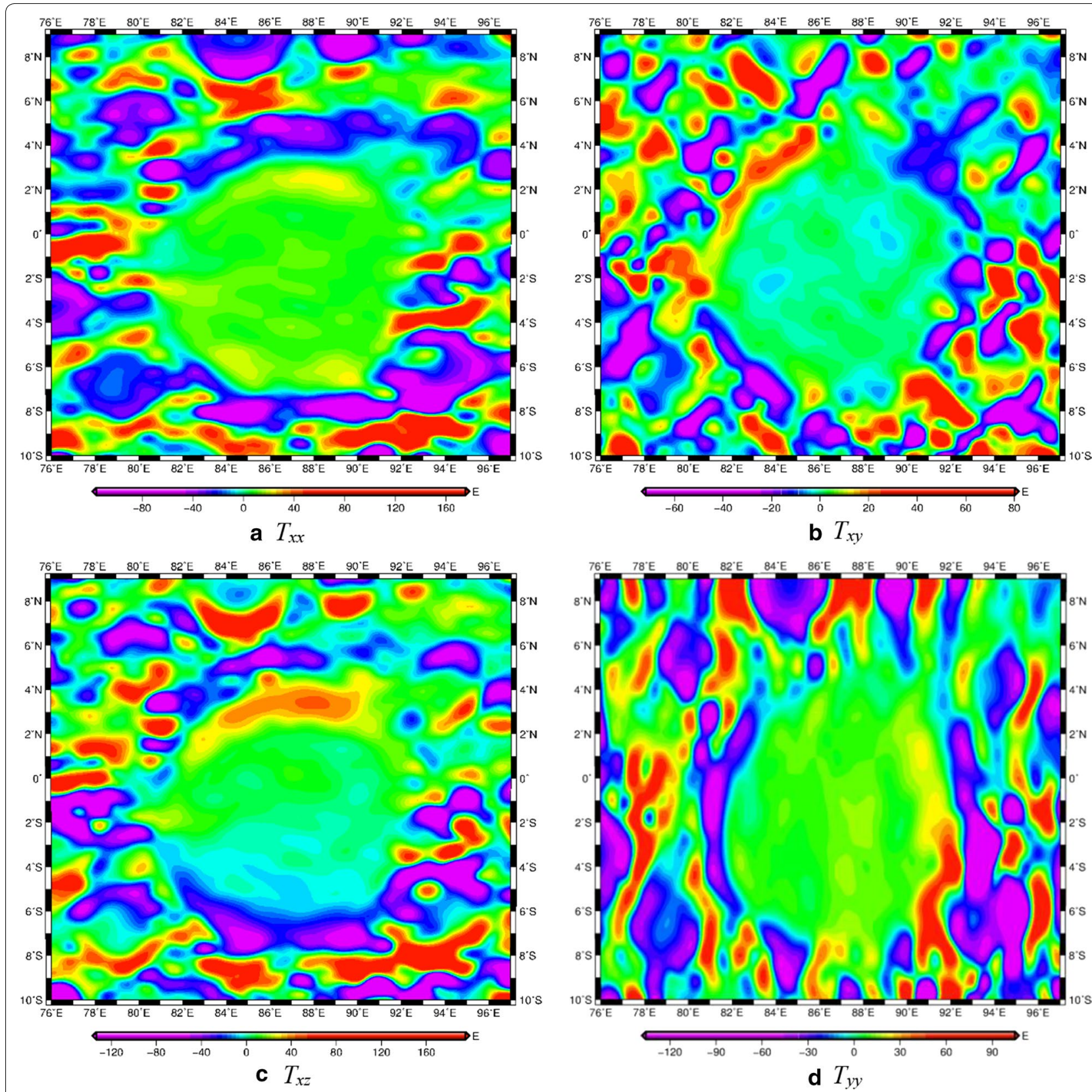

Fig. 14 Lunar GGT datasets derived from GL1500E, a, b, c and $\mathbf{d}$ are $T_{x x} T_{x y} T_{x z}$ and $T_{y y}$ component of the lunar GGT, respectively

section, we will make use of the actual lunar GGT datasets to validate our inversion method.

\section{Inversion of GGT data at lunar mascon}

We selected the Mare Smythii mascon as an example. The Mare Smythii is a lunar mare located along the equator on the easternmost edge of the lunar near side (Wilhelms 1987). According to Liang et al. (2014), our model mesh should have a grid size that approximately equaled to $8 \mathrm{~km}$, i.e., $0.25^{\circ}$ by $0.25^{\circ}$ on sphere (Table 2), which meant the suitable maximum degree of the lunar gravity model should be 720 . Hence, the GGT datasets of this mare derived from lunar gravity field GL1500E with a degree and order up to 720 (The Planetary Data System 2016) are shown in Figs. 14, 15, and the expressions for GGT in LNOF we used came from spherical harmonics (Eshagh 2010). We used a Bouguer correction to remove the gravity effect of the lunar topography and deployed a Gaussian averaging function with an averaging radius of $10 \mathrm{~km}$, which matched the size of the divided cells, to 

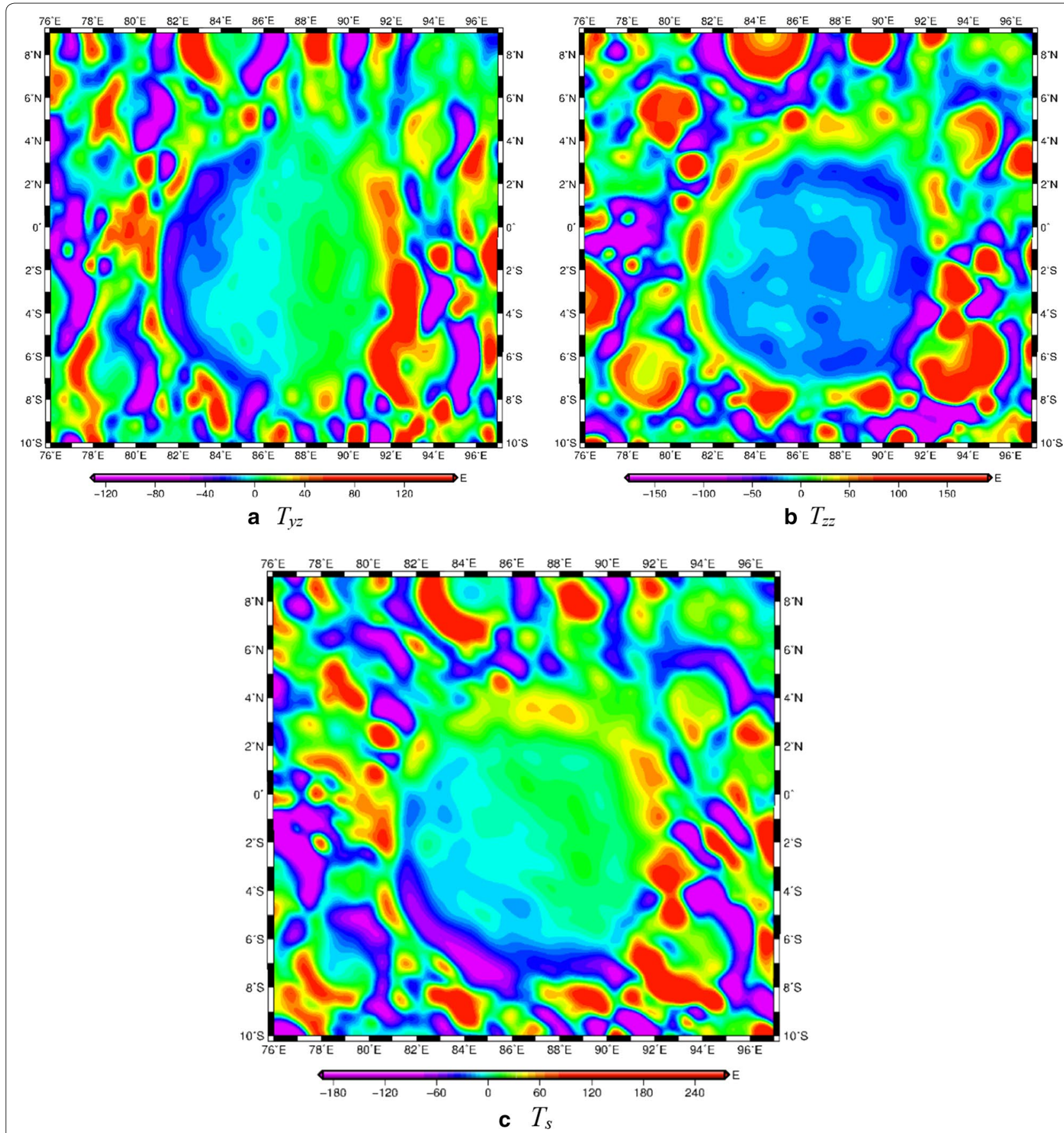

Fig. 15 Lunar GGT datasets derived from GL1500E, $\mathbf{a}$ and $\mathbf{b}$ are $T_{y z}$ and $T_{z z}$ components of the lunar GGT, respectively, and $\mathbf{c} T_{s}$ is the summation of $T_{x x^{\prime}} T_{x y}, T_{x z^{\prime}} T_{y y^{\prime}} T_{y z}$ and $T_{z z}$

reduce the leakage errors caused by high-degree spherical harmonic coefficients. Actually, the Gaussian averaging function was proved to be double-edged (Jekeli 1981); if the selected radius was too large, the filter will remove the useful information and the calculated components will be too smooth. In this paper, we preferred to choose a smaller Gaussian averaging radius which keeps more useful details.

The spherical harmonic coefficients below degree 6 were removed from the GGT datasets we used, and the gravity signal from the left degrees was assumed to be only affected by the lunar crust. The average density of 

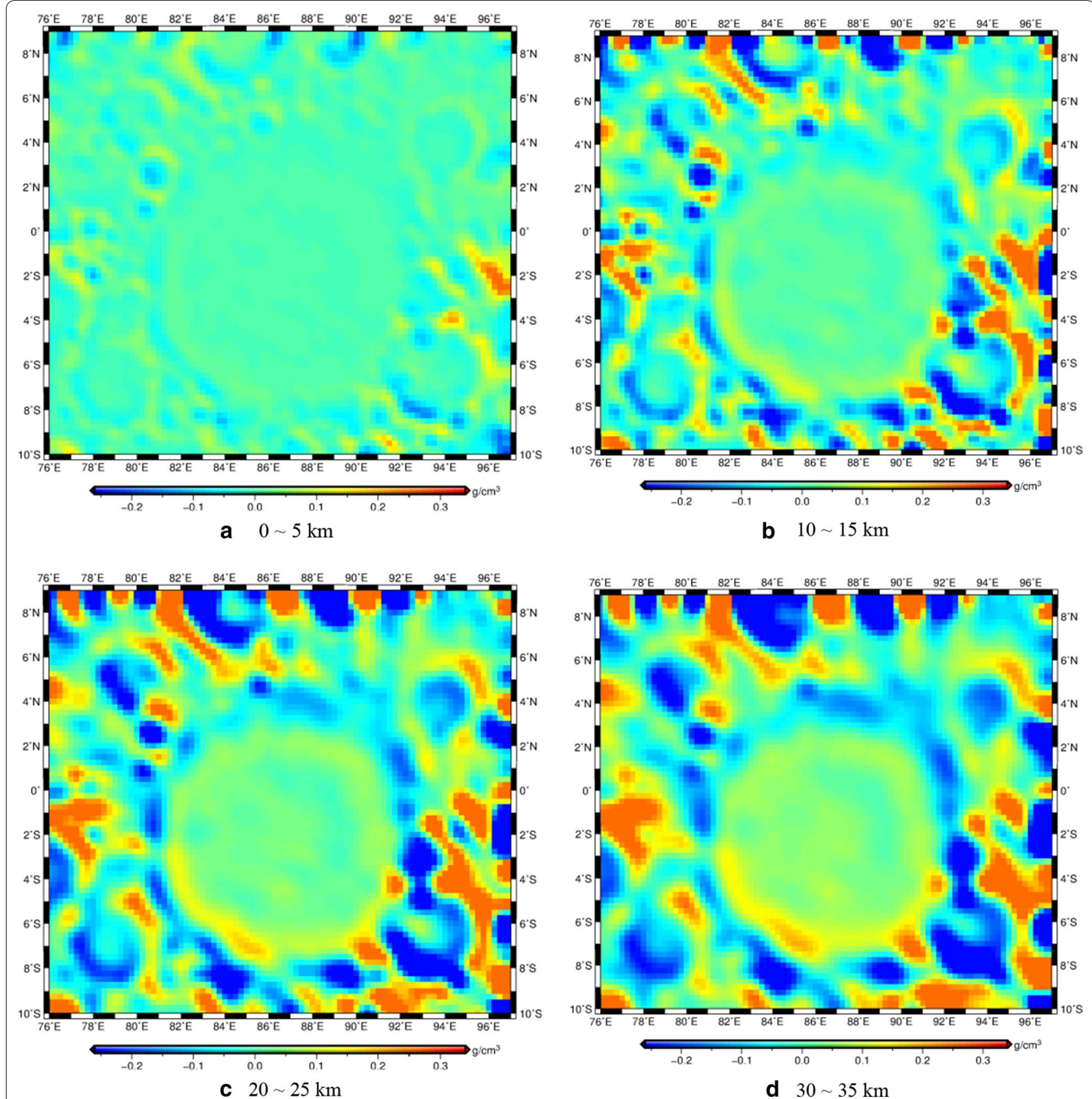

Fig. 16 Relative density distribution for different depths at Mare Smythii area, a, b, c and d are $0 \sim 5 \mathrm{~km}, 10 \sim 15 \mathrm{~km}, 20 \sim 25 \mathrm{~km}$ and $30 \sim 35 \mathrm{~km}$, respectively

the lunar crust we use here is generally $2.56 \mathrm{~g} / \mathrm{cm}^{3}$, at a range of $2.30-2.90 \mathrm{~g} / \mathrm{cm}^{3}$ (Wieczorek et al. 2013). This was also considered as the geological constraint during the inverse process. The 3D mesh and datasets for the inversion of the Mare Smythii are described in Table 2.

By using the GGT datasets as well as the density bounds, we produced an image of the relative density distribution of the Mare Smythii, as shown in Figs. 16, 17 and 18, and the density distribution shows that the depth of Mare Smythii is deeper than which referred from Wieczorek et al. (2013) and similar with the result from Andrews-Hanna et al. (2013).

The gravity gradient reflects the density anomaly; Figs. 16, 17 and 18 shows that there are multi-ring structures (Spudis 1993) at the Mare Smythii site. As the density distribution of the Mare Smythii we extracted 

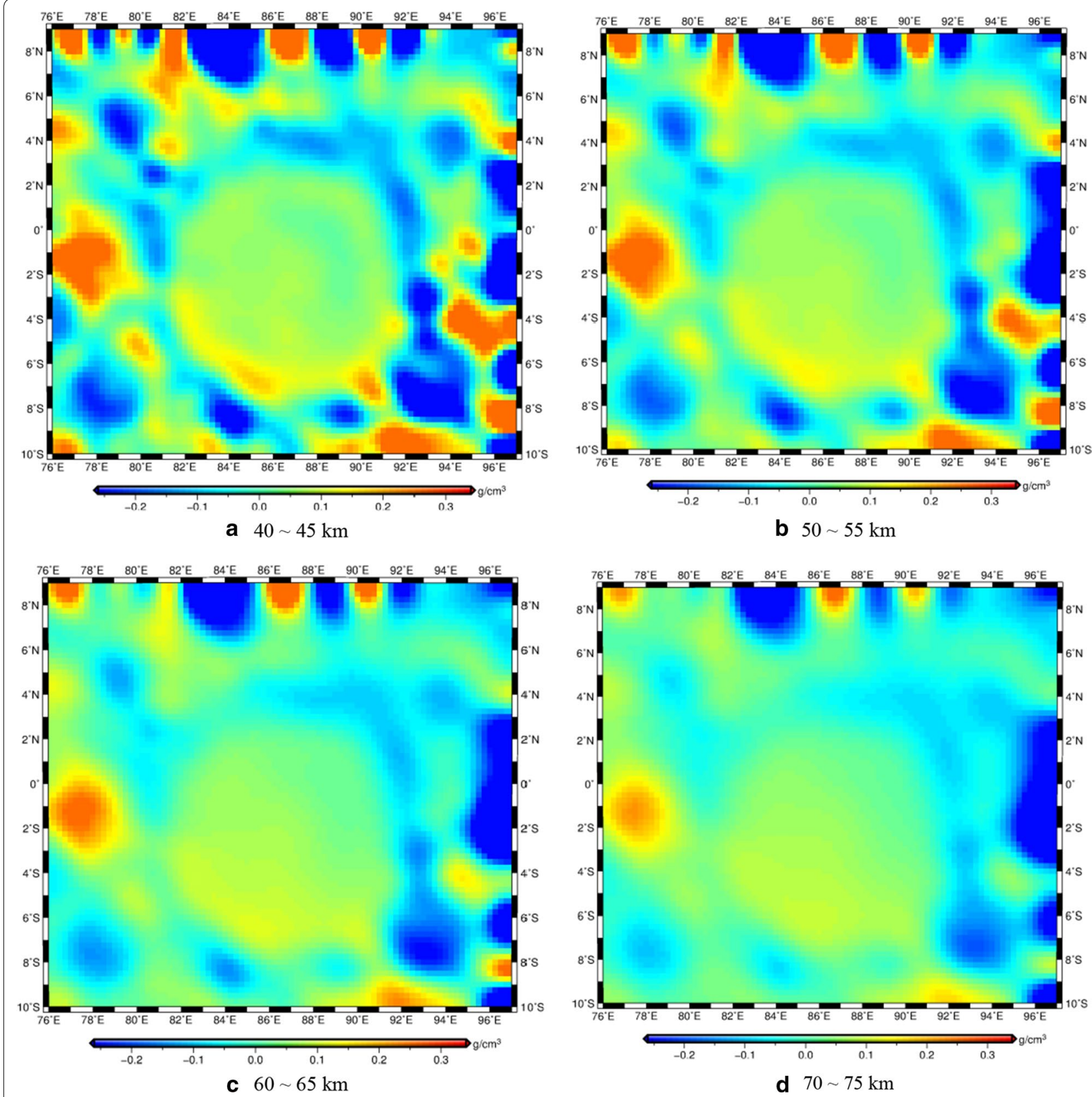

Fig. 17 Relative density distribution for different depths at Mare Smythii area, a, b, c and d are $40 \sim 45 \mathrm{~km}, 50 \sim 55 \mathrm{~km}, 60 \sim 65 \mathrm{~km}$ and $70 \sim 75 \mathrm{~km}$, respectively

through inversion showed some annulus density structures in the shallow stratums and these annulus density structures had the mascon center as the origin point, therefore we infer that there are significant density changes occurred from inside to outside. The formation of these annulus density structures is possibly explained by impacts and crater excavations (Melosh et al. 2013; Montesi 2013). When impacts occur, the kinetic energy is intense and is transformed into heat; this may cause melting and even vaporize the lunar crust, with cracked lunar crust rocks splashing out of the impact basin. After an impact, a rebalancing of the lunar crust and mantle will likely promote an uplifting of the deeper magma to fill in or eject around an impact basin. The density difference as well as the distance of an ejection could be the reason behind the annulus density structures in this area.

The recovered $T_{\mathrm{s}}$ (Fig. 19a) is similar to the original $T_{\mathrm{s}}$ shown in Fig. 15c and the data residual in Fig. 19b, and 

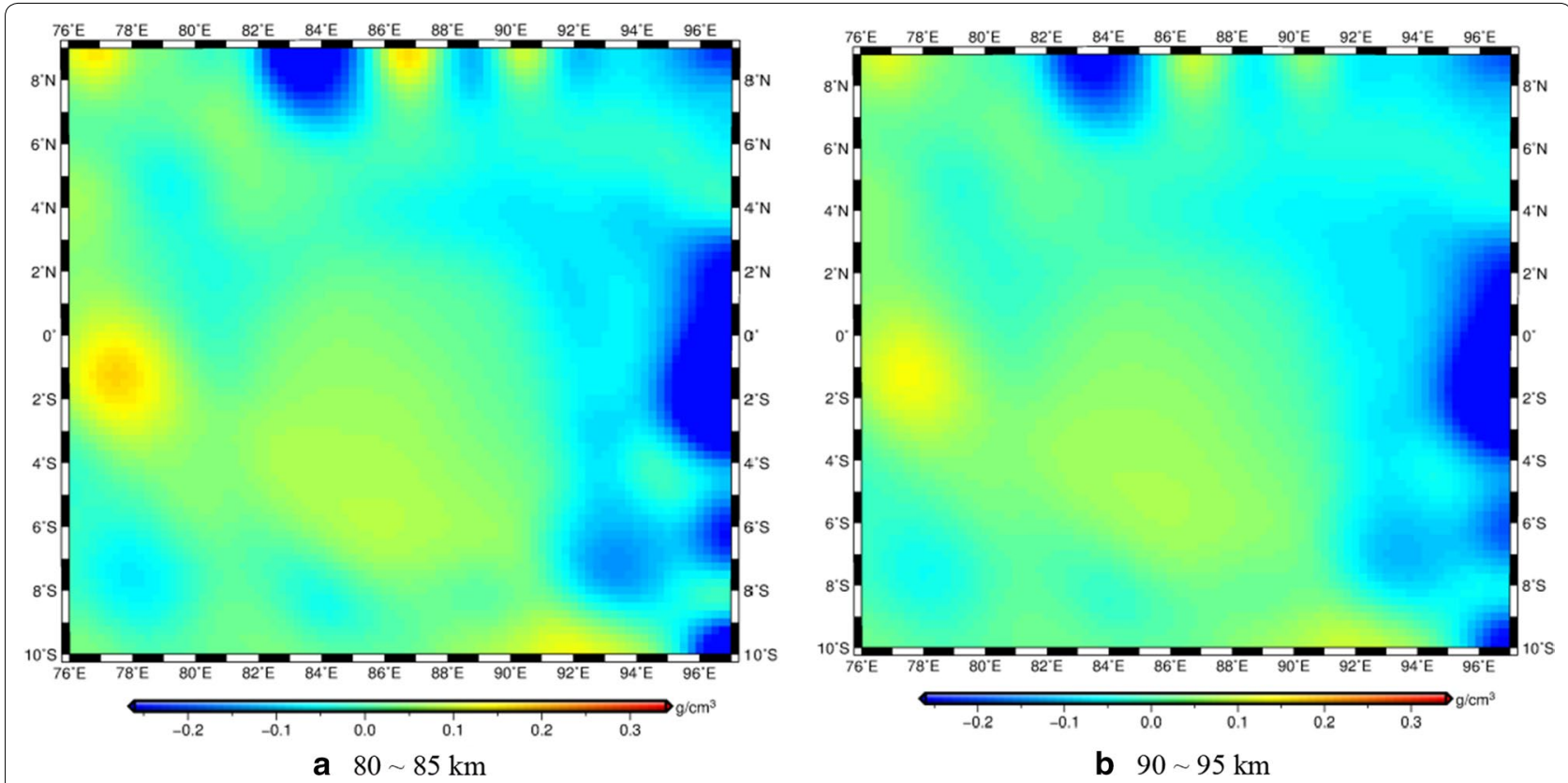

Fig. 18 Relative density distribution for different depths at Mare Smythii area, a and $\mathbf{b}$ are $80 \sim 85 \mathrm{~km}, 90 \sim 95 \mathrm{~km}$, respectively

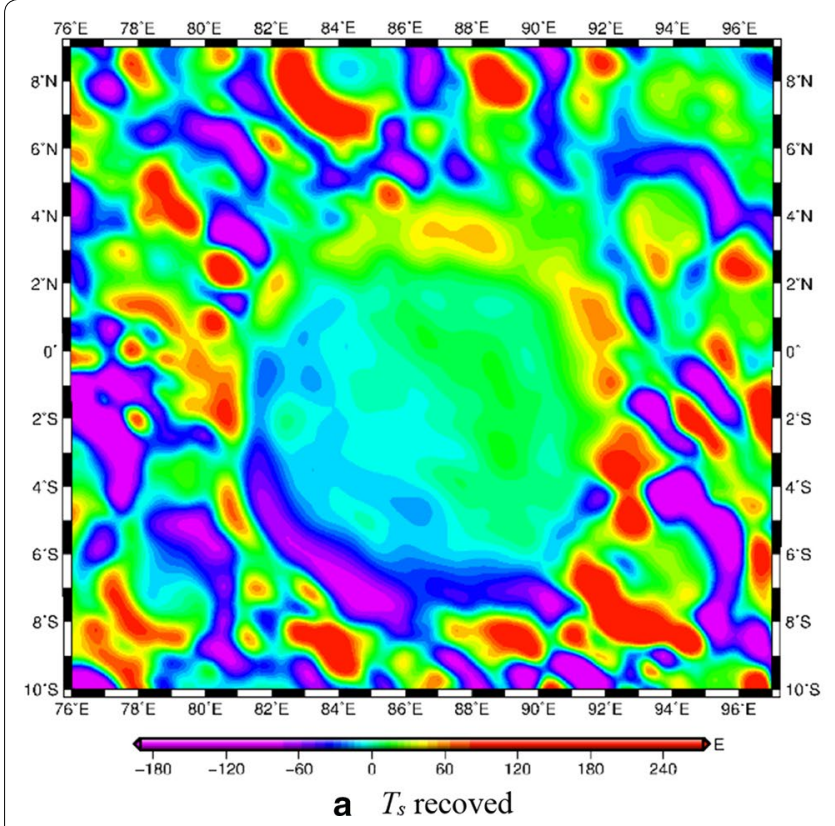

Fig. 19 The recovered $T_{s}(\mathbf{a})$ with residuals $(\mathbf{b})$

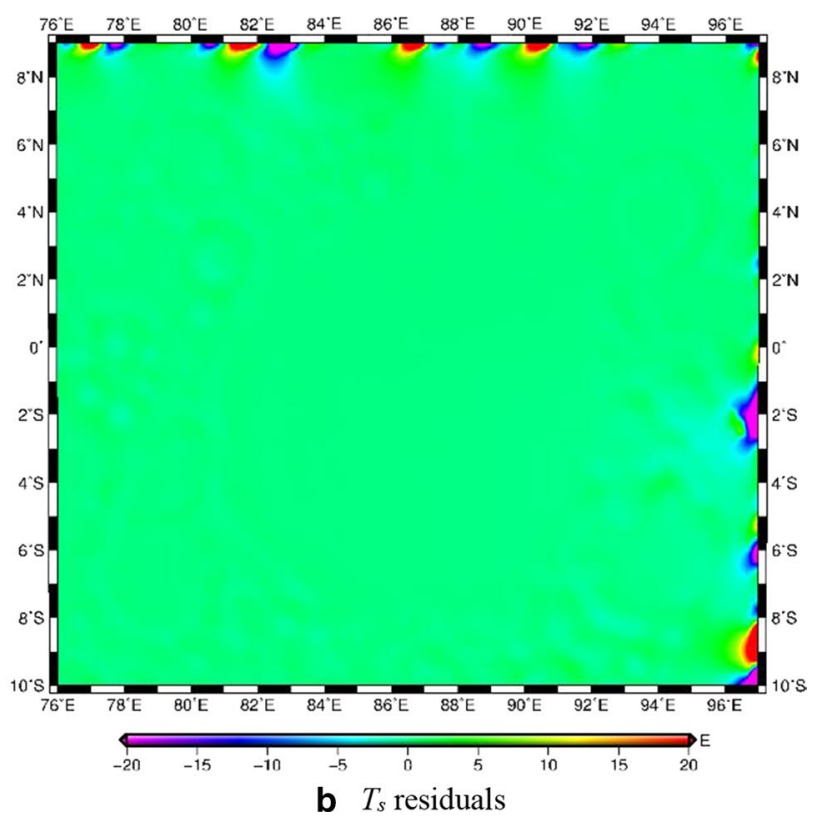

the difference between original $T_{\mathrm{s}}$ and recovered $T_{\mathrm{s}}$ also meets our standard deviation expectation of about $5 \mathrm{E}$. From Fig. 19b, we can see that the residuals inside the Mare Smythii region are close to zero, and it means the good performance of our inversion method. The random big residual values at the verge are caused by the boundary effects.

\section{Conclusion}

An inversion method is fundamental when developing an advanced understanding of the interior structure of planetary geological structures. We presented an effective algorithm that uses full gravity gradient tensor data in spherical coordinate system using local north-oriented frame. In the test case using simulated data, we used two 
different models, single and composite models. By checking the differences between the simulated and recovered models, we demonstrated the reliability of our method. We also tested the method on observed data from the Mare Smythii mascon area located on the lunar near side and obtained its interior density distribution. This method could also be applied more generally in magnetic data inversion and in exploratory studies of geological structures of large areas.

\section{Additional file}

Additional file 1. Length scale in spherical coordinate system.

\section{Abbreviations}

GGT: gravity gradient tensor; SCS: spherical coordinate system; CCS: Cartesian coordinate system; SVD: singular value decomposition; FEM: finite element method; GSF: geocentric spherical frame; LNOF: local north-oriented frame.

\section{Authors' contributions}

YZ and JY conceived and designed the experiment; YZ performed the computation; YW analyzed the data; HW interpreted the results; and JAPR and YQ discussed the results and polished the manuscript. YZ and JY wrote the paper, and all the authors improved it. All authors read and approved the final manuscript.

\section{Author details \\ ${ }^{1}$ Key Laboratory of Earthquake Geodesy, Institute of Seismology, China Earthquake Administration, 48 Hongshan Side Road, Wuhan 430071, Hubei, China. ${ }^{2}$ State Key Laboratory of Engineering in Surveying, Mapping and Remote Sensing, Wuhan University, 129 Luoyu Road, Wuhan 430070, Hubei, China. ${ }^{3}$ MOE Key Laboratory of Fundamental Physical Quantities Measurement, School of Physics, Huazhong University of Science and Technol- ogy, 1037 Luoyu Road, Wuhan 430074, Hubei, China. ${ }^{4}$ Hubei Key Laboratory of Gravitation and Quantum Physics, School of Physics, Huazhong University of Science and Technology, 1037 Luoyu Road, Wuhan 430074, Hubei, China. ${ }^{5}$ Planetary Science Institute, 1700 East Fort Lowell Road, Suite 106, Tucson, AZ 85719-2395, USA. ${ }^{6}$ DFH Satellite Co. LTD, 104 Youyi Road, Beijing 100094, China.}

\section{Acknowledgements}

The authors thank Stephen McClure for fruitful and informative discussions.

\section{Competing interests}

The authors declare that they have no competing interests.

\section{Data and resources}

The source codes for this work can be obtained by contacting the corresponding author. Some of the figures were produced using Generic Mapping Tools (GMT).

\section{Funding}

This work is supported by grant of the National Natural Science Foundation of China (41374024, 41704082), Director Foundation of the Institute of Seismology, China Earthquake Administration (IS201616249), Key Laboratory of Geospace Environment and Geodesy, Ministry of Education, Wuhan University (16-01-02), and Hubei Province Natural Science Foundation innovation group Project (2015CFA011).

\section{Publisher's Note}

Springer Nature remains neutral with regard to jurisdictional claims in published maps and institutional affiliations.

Received: 15 September 2017 Accepted: 27 March 2018

Published online: 16 April 2018

\section{References}

Anderson EG (1976) The effect of topography on solutions of Stokes' problem, Unisurv S-14, Rep, School of Surveying, University of New South Wales, Kensington

Andrews-Hanna JC, Asmar SW, Head JW, Kiefer WS, Konopliv AS, Lemoine FG, Matsuyama I, Mazarico E, McGovern PJ, Melosh HJ, Neumann GA, Nimmo F, Phillips RJ, Smith DE, Solomon SC, Taylor GJ, Wieczorek MA, Williams JG, Zuber MT (2013) Ancient igneous intrusions and early expansion of the Moon revealed by GRAIL gravity gradiometry. Science 339:675-678. https://doi.org/10.1126/science.1231753

Andrews-Hanna JC, Besserer J, Head JW III, Howett CJA, Kiefer WS, Lucey PJ, McGovern PJ, Melosh HJ, Neumann GA, Phillips RJ, Schenk PM, Smith DE, Solomon SC, Zuber MT (2014) Structure and evolution of the lunar Procellarum region as revealed by GRAIL gravity data. Nature 514:68-71. https://doi.org/10.1038/nature13697

Asgharzadeh MF, von Frese RRB, Kim HR, Leftwich TE, Kim JW (2007) Spherical prism gravity effects by Gauss-Legendre quadrature integration. Geophys J Int 169:1-11. https://doi.org/10.1111/j.1365-246X.2007.03214.X

Backus G, Gilbert J (1967) Numerical applications of a formalism for geophysical inverse problems. Geophys J R Astron Soc 13:247-276. https://doi. org/10.1111/j.1365-246X.1967.tb02159.x

Backus G, Gilbert F (1968) The resolving power of gross Earth data. Geophys J R Astron Soc 16:169-205. https://doi.org/10.1111/j.1365-246X.1968. tb00216.x

Backus G, Gilbert F (1970) Uniqueness in the inversion of inaccurate gross Earth data. Philos Trans R Soc Lond Ser A Math Phys Sci 266:123-192

Barbosa V, Silva J (1994) Generalized compact gravity inversion. Geophysics 59:57-68. https://doi.org/10.1190/1.1443534

Bell R, Hansen R (1998) The rise and fall of early oil field technology: the torsion balance gradiometer. Lead Edge 17:81-83. https://doi. org/10.1190/1.1437836

Bouman J, Ebbing J, Fuchs M, Sebera J, Lieb V, Szwillus W, Haagmans R, Novak P (2016) Satellite gravity gradient grids for geophysics. Sci Rep 6:21050. https://doi.org/10.1038/srep21050

Calvetti D, Morigi S, Reichel L, Sgallari F (2000) Tikhonov regularization and the L-curve for large discrete ill-posed problems. J Comput Appl Math 123:423-446. https://doi.org/10.1016/S0377-0427(00)00414-3

Capriotti J, Li Y, Krahenbuhl R (2015) Joint inversion of gravity and gravity gradient data using a binary formulation. In: International workshop and gravity, electrical \& magnetic methods and their applications, Chenghu, China, 19-22 April 2015. Society of Exploration Geophysicists and Chinese Geophysical Society, pp 326-329. https://doi.org/10.1190/ gem2015-085

Eshagh M (2008) Non-singular expressions for the vector and the gradient tensor of gravitation in a geocentric spherical frame. Comput Geosci 34:1762-1768. https://doi.org/10.1016/j.cageo.2008.02.022

Eshagh M (2010) Alternative expressions for gravity gradients in local north-oriented frame and tensor spherical harmonics. Acta Geophys 58:215-243. https://doi.org/10.2478/s11600-009-0048-z

Farquharson CG (2008) Constructing piecewise-constant models in multidimensional minimum-structure inversions. Geophysics 73:K1-K9. https:// doi.org/10.1190/1.2816650

Freed AM, Johnson BC, Blair DM et al (2014) The formation of lunar mascon basins from impact to contemporary form. J Geophys Res (Planets) 119:2378-2397. https://doi.org/10.1002/2014JE004657

Geng M, Yang Q, Huang D (2015) 3D joint inversion of gravity-gradient and borehole gravity data. Explor Geophys 48:151-165. https://doi. org/10.1071/EG15023

Guillen A, Menichetti V (1984) Gravity and magnetic inversion with minimization of a specific functional. Geophysics 49:1354-1360. https://doi. org/10.1190/1.1441761

Heck B, Seitz K (2007) A comparison of the tesseroid, prism and point-mass approaches for mass reductions in gravity field modeling. J Geod 81:121-136. https://doi.org/10.1007/s00190-006-0094-0

Jansen JC, Andrews-Hanna JC, Li Y et al (2017) Small-scale density variations in the lunar crust revealed by GRAIL. Icarus 291:107-123. https://doi. org/10.1016/j.icarus.2017.03.017

Jekeli C (1981) Alternative methods to smooth the Earth's gravity field. NASA technical report

Last B, Kubik K (1983) Compact gravity inversion. Geophysics 48:713-721. https://doi.org/10.1190/1.1441501 
LiY (2001) 3-D inversion of gravity gradiometer data. In SEG technical program expanded abstracts 2001; SEG technical program expanded abstracts; society of exploration geophysicists, pp 1470-1473

Li Y, Oldenburg D (1996) 3-D inversion of magnetic data. Geophysics 61:394408. https://doi.org/10.1190/1.1443968

Li Y, Oldenburg D (1998) 3-D inversion of gravity data. Geophysics 63:109-119. https://doi.org/10.1190/1.1444302

Liang Q, Chen C, Li Y (2014) 3-D inversion of gravity data in spherical coordinates with application to the GRAlL data. J Geophys Res (Planets) 119:1359-1373. https://doi.org/10.1002/2014JE004626

Martinez C, Li Y, Krahenbuhl R, Braga MA (2012) 3D inversion of airborne gravity gradiometry data in mineral exploration: a case study in the Quadrilátero Ferrífero, Brazil. Geophysics 78:B1-B11. https://doi.org/10.1190/ geo2012-0106.1

Matsumoto K, Goossens S, Ishihara Y et al (2010) An improved lunar gravity field model from SELENE and historical tracking data: revealing the farside gravity feature. J Geophys Res. https://doi.org/10.1029/2009JE003499

Melosh HJ, Freed AM, Johnson BC et al (2013) The origin of Lunar mascon basins. Science 340:1552-1555. https://doi.org/10.1126/science.1235768

Meng Z (2016) 3D inversion of full gravity gradient tensor data using SLO sparse recovery. J Appl Geophys 127:112-128. https://doi.org/10.1016/j. jappgeo.2016.02.010

Montesi LGJ (2013) Solving the mascon mystery. Science 340:1535-1536. https://doi.org/10.1126/science.1238099

Muller PM, Sjogren WL (1968) Mascons: Lunar mass concentrations. Science 161:680-684. https://doi.org/10.1126/science.161.3842.680

Oldenburg D, LiY (2005) Inversion for applied geophysics: a tutorial. In: Nearsurface geophysics. Society of exploration geophysicists, pp 89-150

Oliveira VC, Barbosa VCF (2013) 3-D radial gravity gradient inversion. Geophy J Int 195(2):883-902

Paoletti V, Fedi M, Italiano F, Florio G, lalongo S (2016) Inversion of gravity gradient tensor data: does it provide better resolution? Geophys J Int 205:192-202. https://doi.org/10.1093/gji/ggw003

Pedersen LB, Rasmussen TM (1990) The gradient tensor of potential field anomalies: some implications on data collection and data processing of maps. Geophysics 55:1558-1566. https://doi.org/10.1190/1.1442807

Petrovskaya MS, Vershkov AN (2006) Non-singular expressions for the gravity gradients in the local north-oriented and orbital reference frames. J Geod 80:117-127. https://doi.org/10.1007/s00190-006-0031-2

Pilkington M (2012) Analysis of gravity gradiometer inverse problems using optimal design measures. Geophysics 77:G25-G31. https://doi. org/10.1190/geo2011-0317.1

Pilkington M (2013) Evaluating the utility of gravity gradient tensor components. Geophysics 79:G1-G14. https://doi.org/10.1190/geo2013-0130.1

Reed GB (1973) Application of kinematical geodesy for determining the short wave length components of the gravity field by satellite gradiometry, Ohio State University

Spudis PD (1993) The geology of multi-ring impact basins. Cambridge University Press
Sun J, Li Y (2014) Adaptive Lp inversion for simultaneous recovery of both blocky and smooth features in a geophysical model. Geophys I Int 197:882-899. https://doi.org/10.1093/gji/ggu067

The Planetary Data System (2016) GRAIL Moon LGRS derived gravity science data products $\mathrm{V} 1.0$

Tikhonov AN, Arsenin VY (1977) Solution of ill-posed problems. W. H. Winston \& Sons Inc, Washington

Uieda L, Barbosa VCF (2012) Robust 3D gravity gradient inversion by planting anomalous densities. Geophysics 77:G55-G66. https://doi.org/10.1190/ geo2011-0388.1

Vasco DW (1989) Resolution and variance operators of gravity and gravity gradiometry. Geophysics 54:889-899. https://doi.org/10.1190/1.1442717

Vasco D, Taylor C (1991) Inversion of airborne gravity gradient data, southwestern Oklahoma. Geophysics 56:90-101. https://doi.org/10.1190/1.1442961

Wang X, Liang Q, Chao C et al (2015) Crust-mantle structure beneath the sinus iridum mare imbrium basin of the Moon. Earth Sci 40:1566-1575. https:// doi.org/10.3799/dqkx.2015.141

Wieczorek MA, Neumann GA, Nimmo F, Kiefer WS, Taylor GJ, Melosh HJ, Phillips RJ, Solomon SC, Andrews-Hanna JC, Asmar SW, Konopliv AS, Lemoine FG, Smith DE, Watkins MM, Williams JG, Zuber MT (2013) The crust of the Moon as seen by GRAlL. Science 339:671-675. https://doi.org/10.1126/ science.1231530

Wilhelms DE (1987) Geologic history of the Moon, US Geological Survey professional paper 1348

Williams NC (2008) Geologically-constrained UBC-GIF gravity and magnetic inversions with examples from the Agnew-Wiluna greenstone belt, Western Australia. University of British Columbia. https://doi. org/10.14288/1.0052390

Yan J, Goossens S, Matsumoto K et al (2012) CEGM02: an improved lunar gravity model using Chang'E-1 orbital tracking data. Planet Space Sci 62:1-9. https://doi.org/10.1016/.jpss.2011.11.010

Zhang Y, Yan J, Li F, Chen C, Mei B, Jin S, Dohm JH (2015) A new bound constraints method for 3-D potential field data inversion using Lagrangian multipliers. Geophys J Int 201:267-275. https://doi.org/10.1093/gji/ ggv016

Zhdanov MS, Ellis R, Mukherjee S (2004) Three-dimensional regularized focusing inversion of gravity gradient tensor component data. Geophysics 69:925-937. https://doi.org/10.1190/1.1778236

Zuber MT, Smith DE, Lehman DH, Hoffman TL, Asmar SW, Watkins MM (2013a) Gravity Recovery and Interior Laboratory (GRAlL): mapping the lunar interior from crust to core. Space Sci Rev 178:3-24. https://doi.org/10.1007/ s11214-012-9952-7

Zuber MT, Smith DE, Watkins MM, Asmar SW, Konopliv AS, Lemoine FG, Melosh HJ, Neumann GA, Phillips RJ, Solomon SC, Wieczorek MA, Williams JG, Goossens SJ, Kruizinga G, Mazarico E, Park RS, Yuan DN (2013b) Gravity Field of the Moon from the Gravity Recovery and Interior Laboratory (GRAIL) mission. Science 339:668-671. https://doi.org/10.1126/ science. 1231507

\section{Submit your manuscript to a SpringerOpen ${ }^{\circ}$ journal and benefit from:}

- Convenient online submission

- Rigorous peer review

- Open access: articles freely available online

- High visibility within the field

- Retaining the copyright to your article

Submit your next manuscript at $\boldsymbol{\nabla}$ springeropen.com 